\title{
Comparative mapping of crawling-cell morphodynamics in deep learning-based feature space
}

Daisuke Imoto ${ }^{1}$, Nen Saito ${ }^{2,7}$, Akihiko Nakajima ${ }^{1,3}$, Gen Honda ${ }^{1}$, Motohiko Ishida ${ }^{1}$, Toyoko Sugita ${ }^{1}$,

${ }^{1}$ Department of Basic Science, Graduate School of Arts and Sciences, University of Tokyo, Meguro-ku,

8 Tokyo 153-8902, Japan.

$9 \quad{ }^{2}$ Universal Biological Institute, University of Tokyo, Bunkyo-ku, Tokyo 113-0033, Japan

$10{ }^{3}$ Research Center for Complex Systems Biology, Graduate School of Arts and Sciences, University of

11 Tokyo, Meguro-ku, Tokyo 153-8902, Japan

$12{ }^{4}$ Department of Biology, Graduate School of Science, University of Tokyo, Bunkyo-ku, Tokyo 113-

13 0033, Japan

$14{ }^{5}$ School of Science, Kitasato University, Kitasato, Sagamihara, Kanagawa 252-0344, Japan

$15{ }^{6}$ Faculty of Science, Yamaguchi University, Yamaguchi 753-8512, Japan

$16{ }^{7}$ Exploratory Research Center on Life and Living Systems, National Institutes of Natural Sciences,

17 Okazaki, Aichi 444-8787, Japan

18 *Corresponding author: cssawai@mail.ecc.c.u-tokyo.ac.jp

Abstract

22 Navigation of fast migrating cells such as amoeba Dictyostelium and immune cells are tightly associated

23 with their morphologies that range from steady polarized forms that support high directionality to those

24 more complex and variable when making frequent turns. Model simulations are essential for quantitative

25 understanding of these features and their origins, however systematic comparisons with real data are

26 underdeveloped. Here, by employing deep-learning-based feature extraction combined with phase-field

27 modeling framework, we show that a low dimensional feature space for 2D migrating cell morphologies

28 obtained from the shape stereotype of keratocytes, Dictyostelium and neutrophils can be fully mapped by

29 interlinked signaling network of cell-polarization and protrusion dynamics. Our analysis links the data-

30 driven shape analysis to the underlying causalities by identifying key parameters critical for migratory

31 morphologies both normal and aberrant under genetic and pharmacological perturbations. The results

32 underscore the importance of deciphering self-organizing states and their interplay when characterizing

33 morphological phenotypes. 


\section{$34 \quad$ Introduction}

35 Cell migration is a fundamental cellular process that underlies embryonic development, wound healing,

36 immunological surveillance and cancer metastasis. In particular, fast migrating cells such as Dictyostelium

37 and migrating immune cells are versatile in their patterns of movement that ranges from random

38 exploratory movements with frequent turns to more persistent migration in a straight path. Dictyostelium

39 cells exhibit random migration ( 1 ) as a phagocyte, in addition to more persistent migration as they

40 aggregate under starvation to form a fruiting body. Exploratory interstitial migration in leukocytes $(2,3)$

41 underlies antigen search and immune surveillance (4), and some are also known to move in a straight line

42 (5). Frequency of cell turning and their angles is dictated by when and where branched networks of F-

43 actin that drives formation of lateral protrusions called pseudopods occur. In Dictyostelium, pseudopods

44 appear arm-like, and their formation and splitting randomizes cell orientation (6). Selective maintenance

45 of pseudopods thus provides directional bias in the shallow attractant gradients (7). Similar F-actin

46 enriched projections in immune cells vary in their appearance from those that are finger-like in DC cells

47 to those more lamellar in neutrophils, however their role in directional choice appears to be conserved $(8$,

48 9). On the other hand, ability to move in a straight and persistent manner requires cell polarity which

49 refers to a long-term state having a dominant leading edge enriched in branched F-actin meshwork and a

50 trailing end with crosslinked actomyosin. In certain cells under geometrical confinement, buildup of

51 hydrostatic pressure by contractility can rapidly switch the protrusion to a bleb which is devoid of F-actin

52 (10). Besides such cases, F-actin driven leading edge protrusion and rear contraction are concomitant in

53 the polarized cells. While the particular shape that cells take depends on the extracellular conditions such

54 as cell-substrate adhesion and diffusible attractants, shapes with broken-symmetry emerge in the absence

55 of extracellular asymmetries and thus their origins are cell-intrinsic by nature $(6,7,11)$. The fact that

56 movement of fast migrating cells depend highly on self-deformation contrasts highly to those of

57 mesenchymal cells such as fibroblasts, which move at an order of magnitude slower speed and are strongly

58 dictated by the asymmetries introduced by the adhesive foci.

60 The common and recurring shapes observed under highly divergent culture conditions and across

61 evolutionary distant species and taxa (12) suggest generality of the self-deforming dynamics in fast

62 migrating cells. Transmigrating neutrophils, genetically or pharmacologically perturbed Dictyostelium

$63(13,14)$ and certain cancer cells $(15)$ take a canoe-like polarized morphology similar to fish-keratocytes

64 and some protozoan amoebae. Conversely, polarized neutrophils under certain genetic and

65 pharmacological perturbations are known to exhibit increased number of pseudopods $(16,17)$. These

66 common and interconvertible morphologies suggest that they reflect basic self-organizing states of motile 
67 cells that can be quantified and compared with minimal reference to the details of the molecular

68 underpinnings and the permissive extracellular conditions. Due to compounding levels of complexity,

69 quantitative characterization of these canonical morphologies also requires one to leave aside fine-scale

70 protrusions such as filopodia and endocytic cups and apply an appropriate coarse-grained description at

71 the cellular-level. The aspect ratio of fish keratocytes was identified as the major variation in shape

72 features by principle component analysis (PCA) (18). Variation in more complex features requires other

73 non-trivial measures of characterization. Fourier and related spectral analysis allows one to extract the

74 periodicity in the protrusion-retraction cycle as well as in their spatial ordering (19). Combined with PCA,

75 Fourier description of Dictyostelium cell shape has shown that the morphologies observed at various

76 steepness of a chemo-attractant gradient can be characterized in a two-dimensional feature space that

77 represents differences in degree of elongation, splitting and polarization (20). Zernike polynomials in

78 combination with PCA has been used to classify invasive cancer morphologies in two-dimensional feature

79 space (21). Besides Fourier-based analysis, methods such as tracking of local curvature (22) and

80 pseudopods at the cell edge $(7,23)$ have been employed to characterize spatio-temporal dynamics of

81 membrane protrusions. In RNAi screen of Drosophila culture cells, a large body of hand-picked

82 morphology features has been employed to train a classifier by shallow neural networks(24) and Support

83 Vector Machine (25). These studies indicate that the states of physically realizable morphologies are

84 confined to a relatively low-dimensional feature space (25). The downside of data-driven approaches,

85 however, are that the analysis often remains in a black-box making it difficult for one to understand data

86 with reference to the underlying causalities.

88 A great challenge remains as to how one can quantitatively relate the characterized shapes to the 89 underlying dynamics and vice versa $(12,26,27)$. For the most basic analysis, it is instructive to formulate 90 a top-down model for isolated cells that is free of extracellular context (12), as behaviors under complex 91 environments may later be deduced, given the repertoire of realizable dynamics, from spatially 92 asymmetries and constraints in the key parameters. In the "graded radial extension model", a polarized 93 morphology similar to that observed in fish keratocytes and neutrophils is described without reference to 94 the underlying mechanism by assuming that the plasma membrane extends radially and that its magnitude 95 is spatially graded along the anterior-posterior axis (28). Such a steady and graded distribution is thought 96 to result from reaction-diffusion based symmetry breaking in the activity of the polarity signals GTPases 97 Rac and RhoA at the plasma membrane that specifies the state of F-actin at each given place and time. 98 Resource limitation that prevents one state from dominating the other is expected when the sum of the 99 inactive and active form of the small GTPases is approximately fixed in time (29). Bi-stable reaction- 
100 diffusion systems with the above constraint are known to support a protrusive membrane region (front)

101 and contractile membrane region (rear) to co-exist in a spatially separate domains within a cell - a

102 mathematical manifestation of a stable polarized cell shape (29). On the other hand, pseudopods are

103 transient structures regulated by locally amplified formation of branched F-actin networks. In

104 Dictyostelium this is governed by transient activation in Ras/Rap and PI3K (30), and in case of neutrophils,

105 by Cdc42 and PI3K $(31,32)$. Because the localized protrusive dynamics occur under uniform conditions,

106 they are thought to arise by noise amplification by excitable regulatory network $(23,30,32,33)$. Cdc42

107 in neutrophils and Ras/Rap in Dictyostelium are also known to act positively to strengthen Rac and Rho

108 and hence cell polarity $(16,34-36)$. Mathematical models of random turning and persistent migration

109 thusfar have descried the outcome of either excitability-based $(14,37)$ or polarity-based regulation (38),

110 however realistic shapes resulting from the interplay of cell polarity and pseudopods have not been

111 addressed not to mention the lack of systematic and quantitative morphology comparison between

112 simulations and real data. In this work, to overcome these shortcomings, we first developed a framework

113 that employs deep learning based classifier to obtain objective measures for shape comparison. To train

114 neural networks, we chose the three well-studied fast migrating cells, Dictyostelium, neutrophils and

115 keratocyte which have contrasting degree of cell polarity and pseudopods. The obtained feature space is

116 then analyzed by formulating and computing a model of cell deformation that incorporates a conceptual

117 signaling network based on the current knowledge of the pseudopod and cell polarity dynamics. Our

118 analysis indicates that the proposed dynamics successfully map experimentally observed morphologies

119 across the full range of the feature space and highlights key parameters that define morphologies under

120 normal and aberrant conditions. The present approach provides a general and extendable framework to

121 characterize varieties of other cell shapes in a data-driven manner which can then be interpreted and tested

122 through hypothesis-driven modeling. Provided that there are large sets of simulated timeseries and real

123 data for feature extraction, the ability to help infer the migratory dynamics from snapshot images should

124 also have practical single-cell applications for cell identification.

\section{Results}

129 A feature space related to cell polarity and pseudopod dynamics can be obtained from classification 130 of stereotype morphologies by deep convolutional neural networks

131 For systematic extraction of cell morphology features from microscopy data, a convolutional neural 132 network (Fig. 1a, lower panel) was trained to classify shape of a cell based on whether it exhibited front- 
133 to-tail elongation with 1) a frequent or 2) occasional pseudopods or whether they took 3) a gliding form

134 elongated in the lateral direction. To this end, we used snapshot images of well-studied cell types known

135 for each of the above stereotype morphologies: Dictyostelium (aggregation-stage; 'agg'), neutrophil-like

136 HL-60 and fish keratocytes (Fig. 1a, upper panel; Movie S1). The choice of the reference data was based

137 on the fact that they are well-studied systems and that each represented a different degree of pseudopod

138 formation and cell polarity $(6,12)$. Under our experimental conditions, Dictyostelium cells showed an

139 elongated form in the anterior to posterior direction with locally appearing pseudopods. Fish keratocyte

140 took a canoe-like shape characterized by its long axis orthogonal to the moving direction. HL-60 exhibited

141 an intermediate form between the two where, compared to Dictyostelium, transient protrusions appear less

142 frequently and the overall shape was more horizontally elongated but to a lesser extent than the keratocyte.

143 Image masks of these isolated single cells (Table S1) were normalized in size and orientation (Fig. 1a; see

144 Methods). Hyper-parameters for deep-learning were chosen for relative high-accuracy for various

145 network structures (Materials and Methods). The extracted features were well trained as judged by the

146 high validation accuracy; $97.9 \%$ and $89.7 \%$, for the training and the validation data respectively (fig.

$147 \mathrm{~S} 1 \mathrm{a}, \mathrm{b}$; the mean of the last 10 epochs). The three nodes $\boldsymbol{F}=\left(F_{1}, F_{2}, F_{3}\right)$ that constituted the second to last

148 layer of the network showed good representation of the three data classes: Dictyoselium (fig. S1c; high

$149 F_{1}$ ), HL-60 (fig. S1d; high $F_{2}$ ) and keratocyte (Figure S1e; high $F_{3}$ ), which can further be reduced to two

150 by principal component analysis (PCA). The latency values of PC1, PC2 and PC3 were approximately

$15166.3 \%, 33.5 \%$ and $0.3 \%$, respectively. $\mathrm{PC} 1=(-0.42,-0.35,0.84) \cdot \boldsymbol{F}$ and $\mathrm{PC} 2=(0.76,-0.64,0.11) \cdot \boldsymbol{F}$.

152 Figure $1 \mathrm{~b}$ shows good separation of the three datasets in the PCA space. The keratocyte and Dictyostelium

153 (agg) dataset were found in the high PC1 and high PC2 regions, respectively. The HL-60 dataset were

154 mapped to a low PC1 low PC2 region.

156 The feature metrics acquired above, by the very fact that they constitute a good classifier, should be useful

157 to quantify similarity of cell morphology of one's interest in reference to the trained data. The overall

158 relationship between representative cell contours and the morphology feature is shown in Figure 1c. At

159 first glance, higher PC1 appears to indicate more pronounced elongation in the lateral direction while

160 higher PC2 indicates marked longitudinal elongation and protruding edges. These variations in the feature

161 space not only reflected the average morphology differences between the three training classes but also

162 shape changes in time. Figures $1 \mathrm{~d}-1$ show representative single-cell timeseries from the validation dataset;

163 i.e. a reserved dataset not used for training. Dictyostelium data showed large fluctuations in both the PC1

164 and PC2 direction (Fig. 1d-f), whereas the HL60 (Fig. 1g-i) and keratocyte data (Fig. 1j-1) exhibited more

165 marked changes in the PC1 direction (see also fig. S1c-e for changes in $\boldsymbol{F}$ ). To clarify the relationship 
166 between the PC1-PC2 and the extent of elongation, we re-visited the principle component of the fish

167 keratocyte shape variation reported earlier ('shape mode 1' in (18)). Low aspect-ratio shapes (-2 $\sigma$ in

168 'shape mode 1' (18)) were located near the HL-60 dataset, and high aspect-ratio shapes ( $2 \sigma$ in ' shape

169 mode 1' (18) ) were located near the keratocyte dataset. Consequently, the shape along the 'shape mode

1701 ' of fish keratocyte (18) constitutes a well-confined manifold in the PC1-PC2 space (Fig. 1c; asterisks)

171 to which our fish keratocyte data were also mapped. Furthermore, ellipsoidal shapes with various aspect

172 ratios indicate that an increase in the lateral elongation maps them on the same manifold as the keratocyte

173 dataset (Fig. 1m, PC1 > -1; magenta to pink ellipsoids) while an increase the degree of head-to-tail

174 elongation maps the ellipsoids to another manifold along the PC2 axis (Fig. 1m, green ellipsoids). Rotating

175 these ellipsoids to intermediate angles map these shapes to intermediate PC1 values (fig. S1 f,g).

177 While the analysis of the stretched ellipsoids clearly demonstrated how the cell orientation and the degree

178 of elongation are mapped to the feature space, the Dictyostelium (agg) dataset was markedly offset from

179 these ellipsoids towards negative PC1 values. To further clarify the nature of this deviation, well-defined

180 polygons were subjected to the same feature analysis (Materials and Methods). All 33 geometrical objects

181 tested were mapped within the region of the PC1-PC2 space spanned by the microscopy data (Fig. 1n).

182 Regular polygons and circles were mapped to a low PC1 - PC2 region and their vertically stretched

183 counterparts were mapped to higher PC2 region (Fig. 1n, red). Polygons and ellipsoids that were stretched

184 in the lateral direction marked high PC1 value (Fig. 1n, blue; figure S1h,i). Of particular note were the

185 star objects which scored highest in the PC2 value and deviated markedly in the feature space from the

186 ellipsoids and other objects when stretched (fig. S1i). Stars have higher PC2 compared to squares and

187 triangles indicating that PC2 reflects pointed edges. Comparisons between upright and vertically flipped

188 stars and triangles indicated that degree of pointedness towards the cell front also affects PC2 but in a

189 complex way (Fig. 1n; see also fig. S1j). An analysis of more asymmetrical geometries with varying

190 number of edges showed that they map to a domain in high PC2 with high variations towards negative

191 PC1 values as in the Dictyostelium (agg) dataset; i.e. away from ellipsoidal shapes (Fig. 1o). Rotating

192 the multi-edge forms with the small PC1 values (Fig. 10; 0 to 30 degrees) bring about decrease in PC1

193 (fig. S1k). Further rotation increases PC1 (fig. S1k; 60 to 90 degrees) due to the shape now appearing

194 more horizontally elongated overall. Generality of the results was confirmed in independent real-cell data

195 by analyzing fully differentiated prespore cells of Dictyostelium which is elongated longitudinally and

196 lacks pseudopods thus mapping identically to the elliposoids (fig. S2a, b). Likewise, effector T cells with

197 their signature branching protrusions were broadly distributed along PC2 (fig. S2c, Th1) compared to

198 markedly less polarized regulatory T cells (fig. S2d, Treg). These analysis indicate that our classifier was 
199 able to yield data-driven representation of complex signatures with respect to the cell orientation for both

200 local pseudopodal protrusions and more global cell elongation.

The 'ideal cell' model recapitulates a generalized morphological landscape constrained by the choice of the protrusion speed and the balance between the local protrusion and global polarity. Let us now introduce an ideal cell model (see Equations: Eqs. 1 and 2) that serves as a canonical shape generator of migrating cells that is strictly constrained by the transient protrusion dynamics and polarization (Supplementary Text). To describe the interfacial membrane mechanics, we employed the phase-field method with the addition of an active force $F_{\text {prot }}=a_{\mathrm{w}} W$ (Eq. 1). The model also consists of spatio-temporal dynamics of variable $W$ and variables $U, V$ that define global cell polarity and local protrusions, respectively (Fig. 2a; Eq. 2). These variables are abstract representation of how the respective signaling molecules such as Rac, Rho for $W$ and Ras, Cdc42, PI3K for $V$ are regulated in space and time

212 (Materials and Methods). $W$ is bistable and takes either a low or a high state which signifies the retracting 213 rear and the expanding front, respectively. The dynamics of $W$ therefore supports a polarized shape with $214 W$ being high at one end and low at the other end (Fig. 2b, $t=660 \mathrm{~s}$ ). The low state indicates that $W$ has 215 converted to the other form $W^{*}$ while the integrated sum of $W$ and $W^{*}$ is fixed to $W_{\text {tot. }}$ In addition, there 216 is an excitable network that describes conversion of $U$ to $V$ which is invoked by small signal fluctuations.

217 The important assumption in the model is that these two core networks are coupled so that $V$ promotes 218 conversion of $W$ from the low to the high state, and $W$ catalyzes amplification of $V$ (Fig. 2a). Because $V$ 219 amplifies noisy fluctuations and generates local protrusions through $W$, a leading edge defined by a region 220 with high $W$ is most likely to be perturbed and often split into two (Fig. 2b; $t=700$ ). This is however 221 transient, as $W$ by itself works to maintain global unipolarity hence only one protrusion survives (Fig. 2b; $222 t=800 \mathrm{~s})$. In some cases, a new protrusion can also form away from the anterior and more towards the 223 lateral side and still develop into a new dominant front (Fig. 2c). These features required full 3-variable 224 equations (Eq. 2; Table S3) and were of particular importance in our comparative analysis. Insofar as our 225 parameter search (Table S4 and S5), neither the dynamics of $U$ and $V$ (fig. S3a-d) nor that of $W$ alone (fig. 226 S3e-i) supported these bifurcating protrusions (Supplementary Text). Although our model encompasses

227 the 1- and 2-variable limits which well describe morphologies outside of the training dataset such as oscillatory non-migratory shapes (fig. S3b,c) and non-bifurcating polarized cells (fig. S3f,g), we shall exclude these parameter regimes from the following analysis. Related oscillatory and fan-like morphologies have been addressed earlier (39-41). 
232 In total, 228 parameter conditions (Table S6) were selected by heuristic sampling where we performed

233 grid search in the parameter subspace around hand-picked reference points (fig. S4; Material \& Methods).

234 The advantage of the 'ideal cell' model over the existing models focused on specific cells and conditions

235 is that, it can describe a generalized morphological landscape incurred by the choice of the protrusion

236 speed and the balance between the local protrusion and global polarity. As we shall describe later, this

237 versatility allows us to resolve and characterize cells in different developmental stages and under

238 perturbations. First, of particular interest was whether the cell was elongated in the lateral or anterior-

239 posterior direction. We found $W_{\text {tot }}$ has a large influence in the direction and the degree of overall cell

240 elongation (Fig. 2d; see also Movie S2). For small $W_{\text {tot, }}$ the simulated morphology was near circular (Fig.

$2412 \mathrm{~d}, W_{\mathrm{tot}}=50-70$ ). As $W_{\text {tot }}$ increased, a small region with $W$ appeared, and cells became elongated (Fig. $2 \mathrm{~d}$,

$\left.242 W_{\text {tot }}=80,90\right)$. A further increase in $W_{\text {tot, }}$ expanded the high $W$ region (Fig. 2 d, $W_{\text {tot }}=100$ ) until it

243 encompassed the entire perimeter (Fig. 2d, $W_{\text {tot }}=110$ ). Accordingly, moderately high $W_{\text {tot }}\left(W_{\text {tot }}=80\right)$

244 supported relatively high $F_{1}$ (indicating Dictyostelium-like shape)(Fig. 2d; bottom panel). In the PC1-PC2

245 space, small $W_{\text {tot }}$ yielded low PC1 and low PC2, whereas at a moderately high $W_{\text {tot, }}$ PC2 took high values

246 (Fig. 2e). At high $W_{\text {tot }}, \mathrm{PC} 1$ and PC2 decreased. The overall dependency on $W_{\text {tot }}$ were conserved when

247 other parameters were varied (Fig. 2e; magenta and blue). The other important parameters that affected

248 cell polarity was $\rho$; the conversion rate from $\mathrm{W}$ to $\mathrm{W}^{*}$. High $\rho$ means that the non-zero roots of the cubic

249 equation $-\rho W^{3}+\rho W^{*} W^{2}-W=0$ is large thus supporting a larger domain with high $W$. Therefore, at

250 low $\rho$, the leading edge is small and cells become elongated in the moving direction (fig. S5a; $\rho=4.55$ ).

251 As $\rho$ is increased, leading edge became broader and $F_{3}$ increased (fig. S5b; $\rho=5.56$ ).

253 Occurrence of local protrusions depended largely on $k_{\mathrm{W} 1}$ and $a_{\mathrm{W}}$. $k_{\mathrm{W} 1}$ specifies the depth of the bistable

254 well. For small $k_{\mathrm{W} 1}$, the front-rear asymmetry was weak, and the overall cell shape was near circular (Fig.

$\left.2552 \mathrm{f} ; k_{\mathrm{W} 1}=1.1\right)$. Intermediate $k_{\mathrm{W} 1}$ exhibited mixed dynamics where local protrusions induced asymmetrical

256 deformation however without persistent front-to-back polarity (Fig. 2f; $k_{\mathrm{W} 1}=2.2-55$ ). Large $k_{\mathrm{W} 1}$ elevates

$257 W$ which makes it less affected by the dynamics of $U$ and $V$, and thus supports elongated shape with more

258 marked polar asymmetry in $W$ (Fig. 2f, $k_{\mathrm{W} 1}=110$; Movie S3). Accordingly we obtained high $F_{1}$ (Fig. 2f,

259 lower panel), and PC2 (Fig. 2g). The appearance of local protrusions also depended strongly on the

260 protrusion force $a_{\mathrm{W}}$ (Fig. 2h; Movie S4). For low $a_{\mathrm{W}}$ (Fig. 2h; $a_{\mathrm{W}}=0.8$ ), only small deformation was

261 observed and the overall cell shape was near circular. At an intermediate value of $a_{\mathrm{W}}$ (Fig. 2h; $a_{\mathrm{W}}=1.6$ -

262 2.4), cells were more longitudinally elongated and the cell displacement was more directional. At high

$263 a_{\mathrm{W}}\left(\right.$ Fig. $\left.2 \mathrm{~h} ; a_{\mathrm{W}}=3.2-4.0\right)$, multiple pseudopods appeared, and the cell orientation changed frequently 
264 (Movie S4). There was an increase and a decrease in $F_{1}$ and $F_{2}$ respectively (Fig. $2 \mathrm{~h}$, lower panel). The

265 PC2 score increased accordingly (Fig. 2i).

Parameters that affected the pseudopod dynamics were $\mu$ and $\gamma$ which define the downregulation rate of $V$ and $U$, respectively. Low $\mu$ (Fig. 2 j; $\mu=0.1$; Movie S5) elevates $V$, hence the concomitant increase in $W$ supported a laterally elongated shape. The polarized shape was highly persistent as high $V$ renders the patterning less prone to noise perturbation. On the other hand, at intermediate to high value of $\mu$, cells became more elongated longitudinally and the polarity was less persistent (Fig. $2 \mathrm{j} ; \mu=0.5-0.9$ ) . Here, the high $W$ domain was easily disrupted; fronts frequently split, and the cell orientation was altered (e.g.,

273 a Y-shaped front in Fig. $2 \mathrm{j}$ at $\mu=0.7$ and 0.9 ). Accordingly, $F_{3}$ (Fig. 2j; bottom panel) and the PC1 score

274 decreased at high $\mu$ (Fig.2k). Similarly, at low $\gamma$, pseudopods split frequently and new pseudopods were 275 rare (fig. S5c, d; $\gamma=0.1$ ) and the opposite was true for high $\gamma$ (fig. S5c, d; $\gamma=0.5$ or 0.7 ). Additionally,

276 for splitting to occur, it was important that diffusion of $W$ does not average out the local perturbations.

277 Broad leading edge split at low $D_{\mathrm{W}}$, (fig. S5e ; $D_{\mathrm{W}}=0.6,1.8$ and 2.4), but was sustained at high $D_{\mathrm{W}}$ (fig.

278 S5e; $D_{\mathrm{W}}=3.6$ ). These details only made subtle changes in our morphology feature (fig. S5e,f). The

279 boundary flux $\chi_{\mathrm{U}}$, was also important to restrict the $U-V$ reaction at the edge (fig. S3b,c). Splitting of the

280 front occurred more frequently at high $\chi_{\mathrm{U}}$ (fig. S5g). Due to the temporal nature, the feature vector on

281 average remained almost unchanged (fig. S5h).

283 Morphology-based mapping of model parameters can help infer candidate dynamics

284 The distribution of the simulation data in the PC1-PC2 space were found to span a large region occupied 285 by the training dataset (Fig. 3a, black circles) further vindicating the ability of the ideal cell model to 286 describe the characteristic morphologies. Proximity of the time-averaged simulated morphologies (Fig.

$2873 \mathrm{~b}$ ) to the average of the three reference dataset was analyzed by computing the Euclidean distance in the 288 feature space $\boldsymbol{F}=\left(F_{1}, F_{2}, F_{3}\right)$. According to the reference data, the distance was designated as Score-D 289 (Dictyostelium), Score-H (HL-60), Score-K (keratocytes), and ranked in the ascending order; i.e. a low 290 score means high similarity (Table S7). The time averaged morphology feature in the PC1-PC2 space and 291 the time-series of the top ranking simulations are shown in Fig. 3b (filled circles) and Figures 3c-k, 292 respectively. Simulations with high Score-D on average exhibited morphology that closely resembled 293 the aggregation-stage Dictyostelium with their elongated form in the anterior-posterior direction 294 accompanied by a few pseudopods that frequently reoriented cell directionality (Fig. 3c; Movie S6). 295 Similarly, simulations that ranked high for Score-H (Fig. 3f; Movie S7) exhibited fan-like cell shape that 296 moved directionally with some occasional turning as observed in HL-60. For high Score-K simulations, 
297 cells had canoe-like shape with high directional persistence (Fig. 3i; Movie S $\underline{8}$ ), however the similarity

298 was not as high as the other two dataset. The top ranking parameter sets were found near the median of

299 the reference dataset in PC1-PC2 space except for the keratocyte shape which deviated for the simulation

300 in the PC2 direction. In our parameter search, we were unable to obtain simulation results that mapped

301 closer to the average of the keratocyte data. The overall mapping of real cell data and model simulations

302 were conserved when intermediate layer of the classifier was used to obtain the feature space

303 (Supplementary Text; fig. S6; SI Table 9).

305 Although similarity was evaluated based on still images, dynamics of high ranking simulations were by

306 and large consistent with those of real cells. Both Dictyostelium and HL60 (Fig 4a,b left panels) showed

307 anterior projections (Fig. 4e bottom panel red regions) that bifurcated from time to time and traveled

308 towards the high curvature region at the rear (42). The wave-like appearance was somewhat more

309 prominent in Dictyostelium. In both cell types, the posterior end was characterized by a high curvature

310 region that persisted over time. These dynamical features were well recapitulated in the simulations (Fig.

$3114 \mathrm{a}, \mathrm{b}$ right panels). Moreover, there was a good agreement between the simulations and the real data in the

312 cell trajectories. The mean square displacement (MSD) of the centroid showed a characteristic time-scale

313 dependency where it was proportional to the square of the elapsed time $\left(\Delta T^{2}\right)$ for $\Delta T<\tau_{0}$ (Fig. $4 \mathrm{c}$, magenta

314 line) and to $\Delta T$ for $\Delta T>\tau_{0}$ (time domain, Fig. $4 \mathrm{c}$ red line). In other words, cells moved ballistically i.e.

315 at a constant velocity for $\Delta T<\tau_{0}$ and more like a Brownian particle for $\Delta T>\tau_{0}$. $\tau_{0}$ can be interpreted as

316 the persistence time for directional migration, and square root of the MSD at the inflection point $X_{0}$

317 characterizes the persistence length. Throughout this paper, we chose the time-scale factor $\tau^{\prime}=10$ based

318 on approximate matching in the crossover point of the two regression lines between the top ranking

319 simulations and the real-cell data (Fig. 4c,d; red lines). For the top Score-D simulations, we obtained $\tau_{0}=$

$32087 \mathrm{sec}, X_{0}=9.8 \mathrm{cell}$ length, compared to $\tau_{0}=151 \mathrm{sec}$ and $X_{0}=15.2 \mathrm{cell}-$ length in the real data which are

321 in good agreement with values reported earlier (43). Trajectories of top ranking simulations for Score-H

322 were more persistent $\left(\tau_{0}=257 \mathrm{sec}, X_{0}=22.1\right.$ cell length $)$ as was the case for the real HL60 data $\left(\tau_{0}=\right.$

$323278 \mathrm{sec}, X_{0}=47.7$ cell length).

325 The other important feature of random migration is the relation between pseudopod dynamics and the cell 326 orientation (7). New pseudopods frequently appeared in vacant regions (Fig. 4e left), or on top of a pre327 existing pseudopod thereby giving the cell the appearance of Y-shape ('Y-split' in ref (7)). In other cases, 328 pseudopods continued to extend while turning ('one-way-split' in ref (7)). For aggregation-stage 329 Dictyostelium data, the relative occurrence of de novo formation and splitting of pseudopods was 
330 approximately $47 \%$ and 53\% respectively (Fig. 4f). In high ranking simulations, they were $34 \%$ and

$33166 \%$. Similarly for HL60, de novo formation and splitting was $21 \%$ and $79 \%$ in the top ranking simulation,

332 and in real cell data they were $12 \%$ and $88 \%$ (Fig. $4 \mathrm{~g}$ ). The extension angles relative to the direction of

333 centroid displacement was about $20-40^{\circ}$ for both Dictyostelium and HL60 data and their top-ranking

334 simulation counterparts (Fig. 4h,i). Although there was some overrepresentation of extension angles

335 around $90^{\circ}$ in the simulation, the angles above $120^{\circ}$ were rare in both real data and simulations. All in

336 all, these results demonstrate that the model, albeit its simplification, is able to recapitulate semi-

337 quantitatively both the persistent random walk behavior and the underlying morphology dynamics in

338 Dictyostelium and HL-60 cells.

340 Mapping of morphological diversification in the feature space predicts key parameters for state 341 transition

342 Although the datasets analyzed above showed little overlap with one another in the feature space, it should

343 be noted that these coordinates are by no means singular representation of specific cell-types and species

344 from which the data were obtained. As we saw above, there was a large cell-cell variability in the fish

345 keratocyte data that constituted a distinct manifold in the feature space (Fig. 1b; yellow). Likewise, cell-

346 cell variability was evident in the aggregation-stage Dictyostelium cells along the PC2 axis (Fig. 1b; green).

347 To see how changes in cell-intrinsic properties alter their positions in the feature space, data from new

348 experimental conditions expected to alter cell polarity were studied (Fig. 5a). Undifferentiated

349 (vegetative) Dictyostelium cells took less elongated shape than the aggregation-stage Dictyostelium cells

350 under the same substrate and buffer condition. Their aspect ratio on average was smaller than aggregation-

351 stage Dictyostelium but larger than that of HL-60 (Fig. 5b). Accordingly, in the PC1-PC2 space, the

352 vegetative Dictyostelium was mapped between aggregation-stage Dictyostelium and HL-60 (Fig. 5a;

353 magenta reverse triangles). Model simulations that ranked similar to the vegetative Dictyostelium data

354 (Fig. 5c; Movie S9) had small $a_{\mathrm{w}}$ in common (Table S8). Similarly, we analyzed HL-60 cells treated

355 with microtubule destabilizer nocodazole which is known to strengthen neutrophil cell polarity $(44,45)$.

356 Nocodazole-treated HL-60 cells showed morphology similar to keratocyte with a somewhat smaller aspect

357 ratio (Fig. 5d;) and were mapped between the non-treated HL-60 and the keratocyte datasets (Fig. 5a; blue

358 triangle). The nocodazole-treated HL-60 cells exhibited shape fluctuations making them wobble which

359 was also observed in the simulations (Movie S10). These features were well represented in the respective

360 simulations that ranked high for shape similarity (Fig. 5e). In addition to high $W_{\text {tot }}$, high ranking

361 simulations had relatively low $a_{\mathrm{w}}$ or $k_{\mathrm{w} 1}$ (Table S8). This can be interpreted from the fact that low $a_{\mathrm{w}}$ 
362 prevents a cell from breaking apart at high $W_{\text {tot }}$, while low $k_{\mathrm{w} 1}$ makes the polarized front less pronounced

363 and more sensitive to noise perturbation.

365 Next, a null strain of racE (Dicytostelium RhoA homologue) was chosen for the analysis because of its 366 aberrant cell shape that resembled fish keratocytes (46). Under our experimental condition, relatively 367 undifferentiated $r a c E$ - cells exhibited canoe-like shapes that were similar to the fish keratocyte but more 368 dynamic (Fig. 5f). Small fragmented pieces were observed to occasionally split from high curvature 369 regions (Fig. 5f). These data marked relatively high PC1 and PC2 scores and mapped between the 370 keratocyte and the aggregation-stage Dictyostelium data (Fig. 5a; black square). The top ranking 371 simulations for the racE- data (Table S8; high Score-D ( $r a c E-)$ ) exhibited remarkably similar morphology 372 dynamics characterized by high lateral deformation and occasional fragmentation (Fig. 5g; Movie S11). 373 As expected, there was a large overlap with high Score-K data, leaving only the top 3 Score-K data (Table 374 S7 bottom rows; $\|F\|_{2}<3 \times 10^{4}$ ) that uniquely mapped to the keratocyte data. Compared to either the 375 high Score-D (veg) or the high Score-D (agg) data, the high Score-D (racE-) data had large $W_{\text {tot }}$ (Table 376 S8) consistent with the laterally extended cell shape. All in all, the above analysis suggests key parameters $377 a_{\mathrm{w}}$ and $W_{\text {tot }}$ that are pivotal for the state transition between the characteristic morphologies. This was 378 further verified by studying unexamined regions in the high-dimensional parameter space near the top 379 ranking simulations for vegetative-stage Dictyostelium. Increasing the value of $a_{\mathrm{w}}$ brought the 380 morphology score closer to that of the aggregation-stage cell (Fig. 5h). In contrast, increasing $W_{\text {tot }}$ 381 increased PC1 and brought the shape state closer to the nocodazole-treated HL-60 and racE-cells (Fig. $3825 h)$.

\section{Discussion}

386 In this study, we presented a hybrid cell morphology analysis that combined deep-learning-based 387 extraction of morphological metrics from microscopy data and dynamical model simulations. 388 Convolutional neural network was trained to classify stereotype morphology of migrating cells represented 389 by HL-60, Dictyostelium and fish keratocytes. The feature vector of the trained classifier showed that the 390 three representative morphologies can be described in low dimensional feature space; the first component 391 represents elongation in the lateral direction and the second component represents anterior-posterior 392 elongation and edges. The new feature space essentially expanded the principle shape variations 393 associated with cell polarity previously identified in the keratocyte data (18) to encompass narrowly 394 polarized cell morphologies with pseudopodal extensions. The finding of low dimensional feature space 
395 is in line with an earlier feature representation of Dictyostelium cells based on Fourier-mode

396 decomposition of the cell contour (20). While being less analytically clear than the Fourier analysis, the

397 present approach allows one to distinguish morphology with respect to the cell orientation and thus suited

398 to analyze migratory cells. Interestingly, sample conditions expected to alter cell polarity - vegetative

399 stage Dictyostelium, racE-/Dictyostelium, and the nocodazole treated HL-60 cells were mapped to

400 intermediate coordinates spanned by these two major manifolds. Moreover, the new sample conditions

401 were each found clustered without significant overlaps with the training data sets. These results suggest

402 that the region spanned by the three training datasets (keratocyte, H60, Dictyostelium (agg)) in the PC1-

403 PC2 coordinate constitutes a space continuously occupied by forms realizable by genetic and phenotypic

404 variations.

406 Our signaling network model was able to identify regions in the feature space realizable by fast migrating

407 cells that have not been recapitulated in earlier modeling studies. These include narrowly polarized cell

408 morphology, pseudopods as well as their aberrant forms. Parameters chosen based on the similarity with

409 still images provided morphodynamic basis for the random walk trajectory previously approximated by a

410 particle model (1). The main difference between existing models and the present model is that while

411 previous models were either rule-based dynamics $(37,47)$, excitable and oscillatory dynamics $(14,40,48)$

412 or the wave-pinning dynamics $(38,39)$, our work addressed morphological outcomes of the combination

413 of excitable protrusion and bistable polarization that compete for dominance under limited $W_{\text {tot }}$ i.e. the

414 maximal protrusive activity in addition to a positive feedback regulation between the two. In the model,

415 this is described by the $W$-dependency for the amplification of $V$ (Eq. 2c) and amplification of $W$ by $V$

416 (Eq. 2b). Although how exactly the coupling is implemented biochemically requires further investigation,

417 in neutrophils, $\mathrm{Cdc} 42$ acts globally to enforce cell polarity by promoting actomyosin contractility through

418 its effector WASP in a microtubule dependent manner (49). Recent studies indicate that, in Dictyostelium,

419 Ras at the leading edge interacts with GDP-bound form of RacE to strengthen cell polarity (36). Mapping

420 of the racE- morphology to a high $W_{\text {tot }}$ state (Fig. 5h) hints at the nature of the competition for $W_{\text {tot }}$ in

421 respect to the states of actin: the contractile cortical meshwork that are crosslinked with myosin II and the

422 protrusive dendritic meshwork that requires the Arp2/3 complex for side-branching nucleation. RacE is

423 essential for plasma membrane localization of Diaphanous-related formins (DRFs) (46), and deletion of

424 DRFs (ForA-/ForE-/ForH-) results in the loss of cortical actin. Since the morphology of the null mutant

425 of DRFs phenocopies that of the racE-null cells (46), the increase in $W_{\text {tot }}$ are associated with the absence

426 of DRFs from the plasma membrane. On the other hand, the fluctuating protrusions are largely associated

427 with fast idling pulses of Scar/Wave activities which are amplified by the excitable network (50). Recent 
428 studies suggested that actin nucleators such as formins and Arp2/3 are competing for a limited pool of

429 actin monomers and/or their upstream activators such as Rac-GTP (51-53). Such notion is also supported

430 by an observation that the amount of F-actin is compensated in Scar-/WASP- cells by increased

431 localization of ForH at the cortex (54). Taken together with our mapping of $r a c E$ - data, these observations

432 are in line with our current model view that excitability and cell polarity networks compete for dominance

433 over limited $W_{\text {tot. }}$.

435 The variations in the distinct morphologies of differentiating Dictyostelium cells suggest alterations in the

436 key parameters that serves as a control point. The difference between the vegetative- and aggregation-

437 stage Dictyostelium was ascribed mainly to an increase in the membrane protrusion force $a_{\mathrm{w}}$. The increase

438 in $a_{\mathrm{w}}$ can be understood from the fact that Rac1 (55) and SCAR (56), the essential factors for Arp2/3

439 activation, are known to be expressed at low levels in the vegetative-stage then increase markedly in the

440 aggregation-stage cells. A recent study based on an excitable model (40) suggested a progressive state

441 transition from a circular to amoeboid then to a keratocyte-like shape by the increase in the protrusion

442 force (Fig. 2f. in (40)). Rather, our model predicts that changes in the protrusive force should allow a

443 direct transition from a circular shape (low PC1 low PC2) state to either amoeboid or keratocyte-like form

444 depending on $W_{\text {tot. }}$ Such direct transition has been demonstrated experimentally and was attributed to an

445 increased activity of a nested excitable network (14). However, the elongated shapes in their model were

446 oscillatory and lacked the persistency. The presence of the polarity dynamics underlies the characteristic

447 longitudinally extended cell shapes accompanied by branching pseudopod which do not arise in these

448 models $(14,40)$. Relatively low $D_{\mathrm{w}}$ is required in both vegetative and aggregation-stage Dictyostelium to

449 prevent the polarity dynamics from completely winning over the excitable dynamics. A highly polarized

450 form at high $D_{\mathrm{w}}$; i.e. the 1-variable model limit $\zeta / D \mathrm{w} \rightarrow 0$ (fig. S3e-h; Supplementary Text) is indeed

451 reached by cells that further differentiated into prespore cell-type (fig. S3i). While it is possible that certain

452 cells are in a decoupled state $(\zeta=0)$, the requirement of large $D_{\mathrm{w}}$ for cell polarity signifies the importance

453 of $W$ acting globally. Large $D_{\mathrm{w}}$ may not necessarily be mediated by pure diffusion as the present model

454 postulates, but instead could be realized by other transport processes implicated in cell polarity such as

455 membrane flow or the myosin-II dependent global actin flow. Global actin flow has been shown to

456 maintain asymmetric distribution of de-filamenting factors $(57,58)$, however such global flow may not

457 always be present in polarized cells (59). Since these transport processes are tied to cortical actin, they

458 are naturally accompanied by changes in membrane tension (60) which should also be part of the feedback

459 process from $W$ to $V$. 
462 The present framework of data analysis potentially provides means to test and improve specific models of

463 migrating cells. Distinctions between various excitable $(14,41,61)$ and cell polarity models $(38,62,63)$

464 will become more relevant as we proceed further to analyze detailed geometries and dynamics associated

465 with specific cells and conditions. For example, our ideal cell model gave more frequent rise to 466 pseudopods from the tail region compared to the real cell data. Such a discrepancy could be due to the 467 fact that retraction is assumed to be driven only by the area conservation and that no regulated contractility 468 was explicitly described. While this approximation can be justified when there is reciprocity between the 469 front expansion and the rear contraction as has been shown to hold independently of actomyosin in 470 neutrophils (45), the present model could be modified in the future to include local cortical actomyosin 471 regulation when analyzing detailed shapes of the cell rear and the bleb-based front protrusion. Further 472 improvement of the model and increasing dimensionality of the feature space may work hand in hand with 473 extending the present analysis to classify morphologies exhibited by other cell types of wildtype and 474 mutant backgrounds. For example, the present analysis fails to distinguish the pancake-like shape known 475 for Rac and Rap related mutants that result from uniform expansion (41) and similarly round (i.e. low PC1, 476 low PC2) cells inhibited of actin polymerization. This limit maybe overcome by introducing absolute size 477 instead of normalizing the area so as to distinguish spherical cell versus flattened cell in two-dimensional 478 cell masks. Expanding the analysis to 3-dimensional images would also be better suited to the present 479 machine learning approach. As resolutions and dimensions are increased, the cell-shape based analysis 480 may be supplemented with fluorescence image data of cytoskeletons and their regulators. Given the 481 significant bottleneck in the present simulation by the huge computational loads which required parallel 482 computation by GP-GPU, other avenues of coarse-graining maybe required to extend the present approach 483 to a larger multi-modal analysis.

\section{Materials and Methods}

487 Dictyostelium and HL-60 cell culture and data acquisition. Time-lapse data of freely migrating 488 Dictyostelium, neutrophil-like HL-60, fish keratocyte and differentiated T mouse cells were acquired with 489 an inverted microscope using either 20, 40 or 60x objective lens. For Dictyostelium and HL-60, cells 490 expressing Lifeact fused to $\mathrm{mNeonGreen} \mathrm{(64)} \mathrm{and} \mathrm{mTurquoise2} \mathrm{were} \mathrm{employed,} \mathrm{respectively.} \mathrm{A} \mathrm{Lifeact-}$ 491 mTurquoise2 expression vector was constructed by ligating Lifeact-mTurquoise2 into an episomally 492 replicating plasmid pEBMulti Neo (WAKO, 057-08131) at restriction sites XhoI and NotI. The Lifeact493 mTurquoise 2 expressing stable HL60 cell line was obtained by introducing the plasmid by electroporation 
494 (NEPA21; Nepa Gene, Ltd., Chiba, Japan) followed by G418 selection (1 mg mL $\left.\mathrm{mL}^{-1}\right)$ after 2 days. For

495 fish keratocytes, DIC images were employed. Dictyostelium cells were grown axenically and obtained 496 according to standard protocols as previously described (65). Vegetative Dictyostelium AX4 cells $(\mathrm{N}=$ 4971694 snapshots from 18 timeseries;), aggregation-stage Dictyostelium AX4 cells (starved for 3.5 hours; $498 \mathrm{~N}=2841$ snapshots 19 timeseries;), vegetative LifeactGFP/racE- cells $(\mathrm{N}=330$ snapshots from 8 499 timeseries;) were plated on a non-coated coverglass and images were acquired at $5 \mathrm{sec} /$ frame (aggregation500 stage AX4 and racE- cell) or $15 \mathrm{sec} /$ frame (vegetative AX4). Neutrophil-like HL-60 cells were grown in 501 RPMI1640/glutamate media (Wako 189-02145) supplemented with 12\% FBS (Sigma 172012).

502 Differentiated HL-60 cells were obtained by treating the cells with DMSO for 3 days. Images of HL-60 503 cells on fibronectin-coated glass plates in the presence of $1 \mathrm{nM}$ fMLP $(\mathrm{N}=3468$ snapshots from 23 504 timeseries;) were taken at $5 \mathrm{sec} /$ frame. For nocodazole treatment, differentiated HL-60 cells were 505 collected by centrifugation, suspended in fresh HBSS containing $20 \mu \mathrm{M}$ nocodazole and plated on a 506 coverslip pre-coated with 1-2\% BSA in PBS. Data were acquired within 20-75 min in the same medium 507 in the presence of nocodazole ( $\mathrm{N}=181$ snapshots from 3 timeseries).

Primary cell culture and data acquisition. Keratocytes from the scales of Central American cichlids (Hypsophrys nicaraguensis) were cultured as previously described (66) and images were recorded at 2-s 511 intervals $\left(\mathrm{N}=1590\right.$ snapshots from 12 timeseries;). Naïve $\mathrm{CD} 4^{+} \mathrm{T}$ cells were isolated from the lymph 512 nodes and spleen of C57BL/6 mice by MidiMACS (Miltenyi Biotec). Cells were activated by plate-bound 513 anti-CD3 $\left(5 \mu \mathrm{g} \mathrm{mL}^{-1}\right)$ and anti-CD28 $\left(2.5 \mu \mathrm{g} \mathrm{mL}^{-1}\right)$ with cytokines and blocking antibodies. Th1: $2 \mathrm{ng}$ $514 \mathrm{~mL}^{-1} \mathrm{hIL}-2,5 \mathrm{ng} \mathrm{mL} \mathrm{m}^{-1} \mathrm{mIL}-12$. Treg: $1 \mathrm{ng} \mathrm{mL} \mathrm{m}^{-1} \mathrm{hTGF} \beta, 1 \mu \mathrm{g} \mathrm{mL} \mathrm{L}^{-1}$ anti-IFN $\gamma$. Snapshots from these 515 timelapse recording were employed for feature extraction.

518 Deep-learning-based feature extraction. Mask images were pre-processed as follows (Fig. 1a, left 519 panel): (i) the migration direction was determined from the centroid displacement at a five timeframe 520 interval (equivalent to $25 \mathrm{sec}$ for aggregation-stage and racE-Dictyostelium data, $10 \mathrm{sec}$ for keratocyte 521 data) except for vegetative Dictyostelium data where 1 timeframe (equivalent to $15 \mathrm{sec}$ ) was used, (ii) 522 binarized mask image was rotated to align the migration direction to the y-axis, (iii) the image was rescaled 523 so that the cell area is equal to the area of a circle with 25 pixel diameter. The rescaled masks were each 524 embedded at the center of a blank square frame of $64 \times 64$ pixels. The exact spatial resolution of mask 525 images varied from sample to sample due to rescaling, however they were all in the order of $\sim 0.5 \mu \mathrm{m} /$ pixel. 526 Convolutional neural network (Figure 1a, bottom panel) was implemented using Keras (https://keras.io) 
527 with TensorFlow backend. To make the sample size of the three datasets near equal, data augmentation

528 was performed by rotating the original masks at angles $(< \pm 5 \mathrm{deg})$ randomly picked from a uniform

529 distribution (see Table 1 for the number of samples). Input vectors were processed through layers of

530 convolution operation (Fig. 1a, bottom panel; 'convolution') in addition to layers of max pooling operation

531 with a $3 \times 3$ kernel to render the analysis robust to positional deviation (Fig. 1a, bottom panel; 'pooling').

532 These were then processed through a set of densely connected layers with rectified linear and hyperbolic

533 tangent activation function (Fig. 1a, bottom panel; 'rel' and 'tanh'). In the final layers, the dimension of

534 the vector was reduced to three and were passed to 'softmax' activation function. The values of the three

535 nodes $\left(F_{1}, F_{2}, F_{3}\right)$ before the final softmax layer were employed to represent cell shapes. The number of

536 training epoch was 2000 which was sufficient for adequate learning as determined by the accuracy and

537 loss values (fig. S1a,b).

538

539 Geometrical analysis of feature space. To examine mapping of 2-dimensional geometries in the feature

540 space, 7 well-defined objects were used; circle, isosceles triangle, asymmetric right triangle, rectangle,

541 rhombus, pentagon and star polygon in a specified orientation (Fig. 1n). Triangles, pentagon and star were

542 flipped vertically to obtain total of 11 basic objects. The library was further expanded to 33 shapes by

543 including variants of the basic geometries with different aspect ratio 1:1, 1:2 (Fig. 1n red, horizontally

544 elongated shape to the moving direction) and 2:1 (Fig. 1n blue, flattened shape with vertically elongated

545 to the moving direction).

546

547 Model Equations.

548 To numerically simulate the interface between the plasma membrane and the extracellular space, we

549 employed a phase-field equation in the following form $(38,62,67)$.

$\tau \frac{\partial \phi}{\partial t}=\eta\left(\Delta \phi-\frac{1}{\varepsilon^{2}} G^{\prime}(\phi)\right)-M\left(\int_{\text {cell }} \phi \mathrm{d} r-A_{0}\right)|\nabla \phi|+F_{\text {prot }}(\{W\})|\nabla \phi|$

553 The equation describes the dynamics of a continuous state variable $\phi(r ; t)$ in a two-dimensional space that specifies whether a position $\boldsymbol{r}$ is occupied $(\phi=1)$ or not occupied $(\phi=0)$ by a cell at time $t$. In the present study, we consider initial conditions with a single continuous domain with $\phi=1$. The parameter $\tau$ is the viscous friction coefficient. The first term of the r.h.s represents effective surface tension, where $\Delta \phi$ and $G$ are derived from membrane energy. $G$ is Landau functional describing a bi-stable potential. Here we chose $G(\phi)=18 \phi^{2}(1-\phi)^{2}(62)$. The second term describes restoring force that keeps the cell area close 
560 force with magnitude $F_{\text {prot }}$ that is perpendicular to the boundary $|\nabla \phi| \neq 0$ and thus drives membrane

561 extension. In the present simulations, parameters in Eq. 1 were set so that they are an order of magnitude

562 within generally accepted values (Table S2); surface tension $\eta=1.0[\mathrm{pN}],(68)$, cell area $A_{0}=78.83$

$563\left[\mu \mathrm{m}^{2}\right]\left(\sim 5 \mu \mathrm{m}\right.$ radius circle), protrusive force by actin polymerization $a_{\mathrm{w}} W=0.8-4.0[\mathrm{pN} / \mu \mathrm{m}]$ (for $W \sim$

564 1) (69, 70). Since $\tau$ was not well constrained experimentally and expected to differ between cell-types and

565 the culture conditions, we adopted an empirical value $0.83\left[\mathrm{pN} / \mu \mathrm{m}^{2}\right](67)$, which was then calibrated

566 retrospectively by a multiplier $\tau^{\prime}$ so that the time scale of the simulated cell trajectories match with that of

567 real data as described in the later section. The area constraint $M=0.5 \mathrm{pN} / \mu \mathrm{m}^{3}$ and the size of the boundary

568 layer $\varepsilon=1.0[\mu \mathrm{m}]$ were set close to those in the earlier studies $(62,67)$.

569

570 The 'ideal cell' that can take close to all of the basic phases of morphology features that we have examined

571 (Fig. 1) should consist of two main features: 1) transient appearance of localized protrusions and 2)

572 prolonged presence of single expanding edge and retracting tail to appear under homogeneous

573 extracellular conditions (1). Here, we formulate a reaction-diffusion model that describes these two

574 processes mathematically as follows:

575

$\tau^{\prime} \frac{\partial(\phi U)}{\partial t}=\phi\left(-\frac{\alpha U V W}{K_{k}+\frac{\int d \boldsymbol{r} \phi V W}{\int d \boldsymbol{r} \phi}}+\frac{\beta U V}{K_{p}+\frac{\int d \boldsymbol{r} \phi U}{\int d \boldsymbol{r} \phi}}+s-\gamma U\right)+D_{U} \nabla \cdot(\phi \nabla U)-\phi N(\boldsymbol{r}, t)$

$$
-\chi_{U} \frac{U|\nabla \phi|^{2}}{\int d \boldsymbol{r}|\nabla \phi|^{2}}
$$

$577(2 a)$,

$\tau^{\prime} \frac{\partial(\phi V)}{\partial t}=\phi\left(\frac{\alpha U V W}{K_{k}+\frac{\int d \boldsymbol{r} \phi V W}{\int d \boldsymbol{r} \phi}}-\frac{\beta U V}{K_{p}+\frac{\int d \boldsymbol{r} \phi U}{\int d \boldsymbol{r} \phi}}-\mu V\right)+D_{V} \nabla \cdot(\phi \nabla V)+\phi N(\boldsymbol{r}, t)$

$\tau^{\prime} \frac{\partial(\phi W)}{\partial t}=\phi\left(k_{W 1}\left(-\rho W^{3}+\rho W^{2} W^{*}-W\right)+\zeta V\right)+D_{W} \nabla \cdot(\phi \nabla W)$

582 (2c).

583 where the first two equations for $U$ and $V$ describe an excitable reaction network for transient protrusive

584 dynamics, and the third equation for $W$ describes polarization dynamics, respectively (Fig. 2a;

585 Supplementary Text). The role of the excitable reaction network is to generate transient signals for local 
587 (50). In neutrophils, excitable dynamics of Cdc42 and PI3K activity $(32,33)$ is essential for front

588 protrusions. In Dictostelium, excitable dynamics are observed at the level of spontaneous Ras and PI3K

589 activation $(30,67,71)$. Here, we adopted equations originally introduced to study excitable PI3K activities

590 and the resulting F-actin waves in Dictyostelium (Eq. 2a and 2b) (67). Parameters $\alpha$ and $\beta$ are the rate

591 constants of reaction $U \rightarrow V$ and $V \rightarrow U$ multiplied by the time-scale factor $\tau^{\prime}$. The source of edge

592 fluctuation is introduced as a noise term $\phi N(r, t)(67)$ in Eq. $2 \mathrm{~b}$ which is amplified through $V$ by a positive-

593 feedback described in the first term. Increase in $V$ is then slowed down due to depletion in $U$, and the

594 system eventually recovers the original resting state.

596 The expanding membrane region is determined by $W$ governed by Equation (2c) (Fig. 2a; see

597 Supplementary Text for derivation) which is similar in form to the wave-pinning (29). The same type of

598 equation has been used earlier to study polarized cell shape in fish keratocyte (63) and Dictyostelium (38).

599 The first term describes reaction kinetics with bistability, and the second term describes diffusion. We

600 assume that the sum of $W$ and its reciprocal state $W^{*}$ is conserved ( $W_{\text {tot }}=$ const.) (29) and that $W^{*}$ diffuses

601 much faster than $W$ and thus can be approximated as uniform in space. Hence we obtain

$$
W^{*}=\frac{W_{\mathrm{tot}}-\int \mathrm{d} \boldsymbol{r} \phi W}{\int \mathrm{d} \boldsymbol{r} \phi}
$$

603 Due to the global constraint, this class of bistable system gives rise to coexistence of low and high $W$

604 regions due to stalling of a transitory wave front - a property known as "wave pinning" (29, 63). For the

605 sake of brevity, we shall embed rear retraction passively in the form of restoring force. Thus we set $F_{\text {prot }}$

$606=a_{\mathrm{W}} W$ in Eq. 1 (62) so that the edge expands where $W$ is high, and the rest of the domain with high $W_{\text {tot }}$

$607 / A_{0}-W$ as a result contracts due to area conservation imposed by the second term in Eq. 1 . Note that $W$

608 only specifies where the protrusions and retraction take place and does not assume the origin of their

609 driving force. The variable $W$ can thus be interpreted as an abstract representation of bistable signals such

610 associated with the leading edge of polarize cells as Rac-GTP, or alternatively, $W_{\text {tot }} / A_{0}-W$ with the

611 reciprocal spatial profile to indirectly represent signals that regulate rear contraction such as RhoA-GTP.

612 A recent study has shown that Rac-GTP accumulates at the front of migrating neutrophils regardless of

613 whether it is F-actin filled or blebbing (60). Based on the inherent ability to maintain persistent front-and-

614 back unipolarity, the polarity network (Eq. 2c) serves as a spatio-temporal filter to select or remove local

615 protrusions. This coupling is described by assuming that $W$ positively regulates the positive-feedback

616 amplification of $V$ (Eq. 2c; r.h.s first term) in addition to $W$ feeding back to increase $V$ (Eq. 2a) to reflect

617 F-actin- or tension-dependent positive regulation of leading edge signals in neutrophils and Dictyostelium

$618(60,72,73)$. 
621 Parameter search. Model parameters were selected for the systematic feature analysis (Fig. 3a; Table

622 S6) based on the following criteria: Of the total 22 parameters (Table S2 and S3), eight parameters ( $W_{\text {tot, }}$

$\left.623 a_{\mathrm{W}}, k_{\mathrm{W} 1}, \mu, \gamma, \rho, D_{\mathrm{W}}, \chi_{\mathrm{U}}\right)$ were chosen for detail analysis. Parameters $\left(\alpha, \beta, K_{k}, K_{P}, s\right)$ that define the

624 kinetics of $U, V$ were fixed to those used earlier (67). Two to four independent simulations from different

625 random seeds were executed for a given parameter set to obtain average feature scores $\boldsymbol{F}$. Because grid-

626 search in 8 dimensional parameter space was unattainable due to heavy computational load, we adopted

627 parameter search around manually selected reference parameter sets. First, based on preliminary

628 simulations performed with various parameters, a single parameter set R1 was chosen which gave rise to

629 polarized cell morphology; i.e. elongated shape in the moving direction. Around the reference set R1, the

630 following parameters were varied: $a_{\mathrm{w}}$ and $W_{\text {tot }}$ which appeared to affect the elongation of cell in the moving

631 direction, $\mathcal{X}$ and $D_{\mathrm{W}}$ related to the split of the leading edge, and $\gamma$ that seems to affect the appearance of

632 new pseudopods. We performed a grid search around R1 in 2-dimension $\left(a_{\mathrm{W}}, \gamma\right)$ for $\chi_{\mathrm{U}}=0$ and $\chi_{\mathrm{U}}=50$

633 (fig. S4a left panels) while fixing other 6 parameters. Similarly, a grid search was performed in ( $\left.W_{\text {tot }}, D_{\mathrm{W}}\right)$

634 for $\chi=0$ and 50 (fig. S4a left panels). Second, we chose another parameter set $\mathrm{R} 2$ which gave rise to

635 relatively round shape and random cell displacement (i.e. near HL60-like behavior). Around R2, we

636 varied $\left(a_{\mathrm{W}}, W_{\mathrm{tot}}\right),(\gamma, \rho)$, and $\left(k_{\mathrm{W} 1}, \mu\right)$ at both $\chi_{\mathrm{U}}=0$ and 50 (fig. S4a right panels). Representative results

637 of the simulations are shown in figure S4b and c. Phase diagram of feature vector $\boldsymbol{F}$ around R1 on the $\gamma$ -

$638 a_{\mathrm{W}}$ plane and around $\mathrm{R} 2$ on $\mu-k_{\mathrm{W} 1}$ plane are shown in figure S4d and e, respectively.

641 Pseudopod analysis. For both experiments and simulation data, events of pseudopod formation were

642 detected manually by eye as either 'de novo', 'Y-split' or 'one-way-split' based on ref. (7). New

643 pseudopods that appeared in non-protruding regions (Fig. 4e left) were counted as "de novo

644 formation". Protrusions that appeared close to the leading edge were marked as 'Y-split' because of the

645 resulting cell shape (Fig. $4 \mathrm{e}$ right). When new protrusive events occurred on top of the leading edge so

646 as to offset and steer the extending pseudopod, it was counted as 'one-way-split' (Fig. 4e middle) (7). For

647 the subset of the data, the analysis was performed three independent times to confirm reproducibility. To

648 obtain angular distribution, direction of pseudopod extension 10 seconds from the onset was measured

649 relative to the centroid movement. Aggregation-stage Dicyostelium ( $\mathrm{N}=90$ from 4 timeseries), and the

650 closest simulations ( $\mathrm{N}=44$ from 2 timeseries). HL-60 ( $\mathrm{N}=68$ from 5 timeseries), and the closest

651 simulations $(\mathrm{N}=77$ from 3 timeseries). 
Acknowledgements: The authors thank Shuji Ishihara for discussion and helpful comments. HL-60 cells were obtained from BRC Cell Bank (RCB0041, RIKEN). Lifeact-mTurquoise2 was a gift from Dorus Gadella (Addgene plasmid \#32601). The authors are also grateful to Hiroshi Senoo and Miho Iijima

657 (Johns Hopkins University) for the racE- cells, Jonathan Chubb (University College London) for the

658 Dictyostelium codon optimized NeonGreen, Douwe M. Veltman (University of Groningen) for the 659 Dictyostelium expression vector pDM. This work was funded by grants from Japan Science and 660 Technology Agency (JST) CREST JPMJCR1923, Japan Society for Promotion of Science (JSPS), 661 Ministry of Education, Culture, Sports, Science and Technology (MEXT) KAKENHI JP19H05801 to SS

662 and in part by Joint Research by Exploratory Research Center on Life and Living Systems (ExCELLS)

663 Grant 18-204, MEXT KAKENHI JP19H05416, JP18H04759 and JP16H01442; JSPS KAKENHI JP17H01812 and JP15KT0076 (to S.S.).

\section{Main Figure Legends}

Figure 1. Stereotype migrating cell morphology can be classified in two-dimensional feature space.

670 (a) Representative mask images (upper panels: Dictyoselium (agg; aggregation-stage), HL-60 and fish 671 keratocyte) used for trainining a deep convolutional neural network (lower panel). Masks were normalized 672 in area and aligned downwards in the migrating direction. The feature vector $\boldsymbol{F}=\left(F_{1}, F_{2}, F_{3}\right)$ defined by 673 the three nodes in the last layer was further reduced to PC1 and PC2 by PCA. (b) Mapping of trained data

674 in PC1 and PC2 (dark green + (aggregation-stage Dictyostelium), dark red + (HL-60) and yellow + 675 (keratocyte)). Each data point represents time-averaged scores from a time-series of a single cell. (c) 676 Representative cell contours mapped to the feature space. Asterisks are the first principal shape variation 677 of fish keratocyte (images taken from (18) $(-2 \sigma$ (blue) to $+2 \sigma$ from the mean (purple)). (d-1) 678 Representative time-series and the corresponding feature scores for Dictyostelium (agg) (d-f), HL-60 (g679 i) and fish keratocyte (j-1), respectively. (m-o) Mapping of skewed ellipsoidal shapes (the number 680 indicates aspect ratio) (m), polygons (n) and a multi edge geometry (o) in the feature space. The circled 681 regions in the background $(b, f, i, m, o)$ are 95\% confidence ellipses for the mean of all timeseries 682 combined (dotted) and for the mean of individual cell (filled); green (Dictyostelium), dark red (HL-60), 683 yellow (keratocyte). 
Figure 2. Degree of leading edge expansion and the balance between pseudopod formation and polarity persistence can account for the main morphology feature. (a) Schematics of the dynamical model. Self-amplifying and excitatory synthesis from $U$ to $V(67)$ induces protrusion factor $W$ which can be prolonged by the bistable dynamics (Eq. 2). The reaction takes place in a region $\phi=1$ governed by interface physics according to the phase-field equation (Eq. 1). The membrane expands outward into an unoccupied region $\phi=0$ in the direction perpendicular to the border at a rate proportional to local $W$. Main parameters $\left(\chi_{\mathrm{U}}, \gamma, \mu, k_{\mathrm{W} 1}, \rho, a_{\mathrm{W}}\right)$ are denoted along the associated reaction steps. (b,c) Representative model behavior (overlay plots for $U, V, W$ ); front splitting (b) and pseudopod formation (c). (d-k) Parameter dependence of cell morphology (d,f,h,j upper panels), the average feature $\boldsymbol{F}$ from 2-4 independent simulation runs (d,f,h,j lower panels) and changes in the PC1-PC2 space; (d) $W_{\text {tot }}\left(\chi_{U}=50\right.$, $\left.k_{\mathrm{W} 1}=90, \mu=0.5, \rho=5.5556, \gamma=0.1, a_{\mathrm{W}}=2.4, D_{\mathrm{W}}=3\right)$. (e) Black same as (d), blue $\left(\chi_{\mathrm{U}}=50, k_{\mathrm{W} 1}=100\right.$, $\left.\mu=0.1, \rho=5, \gamma=0.1, a_{\mathrm{W}}=2.4, D_{\mathrm{W}}=3\right)$ and magenta $\left(\chi_{\mathrm{U}}=50, k_{\mathrm{W} 1}=110, \mu=0.5, \rho=4.5455, \gamma=0.1, a_{\mathrm{W}}=4\right.$, $\left.D_{\mathrm{W}}=3\right)$. (f) $k_{\mathrm{w} 1}\left(\chi_{\mathrm{U}}=50, \mu=0.5, \rho=4.5455, \gamma=0.1, a_{\mathrm{W}}=5.6, D_{\mathrm{W}}=3, W_{\text {tot }}=80\right)$. (g) Black same as (f), blue $\left(\chi_{\mathrm{U}}=50, \mu=0.5, \rho=4.5455, \gamma=0.1, a_{\mathrm{W}}=2.4, D_{\mathrm{W}}=3, W_{\text {tot }}=90\right)$, and magenta $\left(\chi_{\mathrm{U}}=50, \mu=0.1, \rho=\right.$ $\left.4.5455, \gamma=0.1, a_{\mathrm{W}}=2.4, D_{\mathrm{W}}=3, W_{\mathrm{tot}}=80\right)$. (h) $a_{\mathrm{W}}\left(\chi_{\mathrm{U}}=30, k_{\mathrm{W} 1}=110, \mu=0.5, \rho=4.5455, \gamma=0.1, D_{\mathrm{W}}\right.$ $=3, W_{\text {tot }}=80$ ). (i) Black same as (h), blue $\left(\chi_{\mathrm{U}}=50, k_{\mathrm{W} 1}=100, \mu=0.1, \rho=5, \gamma=0.1, D_{\mathrm{W}}=3, W_{\text {tot }}=80\right)$. (j) $\mu\left(\chi_{\mathrm{U}}=50, k_{\mathrm{W} 1}=100, \rho=5, \gamma=0.1, a_{\mathrm{W}}=2.4, D_{\mathrm{W}}=3, W_{\text {tot }}=80\right)$. (k) Black same as $(\mathrm{j})$, the blue line is $\left(\chi_{\mathrm{U}}=\right.$ $\left.50, k_{\mathrm{W} 1}=110, \rho=4.5455, \gamma=0.1, a_{\mathrm{W}}=4.8, D_{\mathrm{W}}=3, W_{\mathrm{tot}}=80\right)$.

Figure 3. Mapping of morphology features between simulated and real cell data confirms critical parameters that define the morphology type. (a, b) Mapping of simulated cell morphology from the 228 parameter sets (Grey open circle: subsampled time series (a) and time averages for 2 to 4 independent runs (b). Filled circle: simulations with the highest similarity to aggregation-stage Dictyoselium-rank1 (b, green), HL-60 (b, red) and fish keratocyte (v, orange). (b) The 95\% confidence ellipses of the trained datasets (aggregation-stage Dictyostelium dark green (dark green), HL-60 (dark red) and fish keratocyte (yellow) in Fig. $1 \mathrm{~b}(\mathrm{a}, \mathrm{b})$ are shown for reference. (c-k) Snapshots and the feature scores of the top-ranked simulations; aggregation-stage Dictyostelium (c-e), HL-60 (f-h) and fish keratocyte (i-k).

Figure 4. Consistent exploratory dynamics underlie high similarity in the simulated morphologies.

714 (a,b) Local boundary curvature (top panels) and the protrusion speed (bottom panels) (42) from the real 715 cell data (left panels) and the top ranked simulations (right panels) for Dictyostelium (agg) (a) and HL-60 716 (b). (c,d) Mean square displacement of the centroid of Dictyostelium (agg) (c) and HL-60 (d); real cell 717 data (left panels) and top ranked simulations (right panels). The length scale was normalized by the mean 
718 cell length in the moving direction. (e) Representative snapshots of pseudopod formation; de novo

719 formation (left panel) and splitting of an existing pseudopod (one-way-split (middle panel) and Y-shape

720 (right panel)). (f,g) Fractional occurrence of pseudopods by de novo formation (blue) and splitting

721 (yellow); Dictyostelium (agg) (f) and HL-60 (g). (h-i) Histogram of pseudopod angles obtained from the

722 time-series of real cell data (a-d left panels) and the top ranked simulations for Dictyostelium (agg) (h) and

723 HL-60 (i).

725 Figure 5. Mapping of non-trained morphologies with perturbing cell polarity predicts a continuous

726 landscape along critical parameters. (a) Morphology features of real cell data from Dicyostelium (veg;

727 vegetative stage)(pink inverted triangle), Dicyostelium racE- (red square) and nocodazole-treated HL-60

728 (blue upright triangle) and those from simulations exhibiting highest similarity (filled circles). Colored

729 regions in the background represent the reference microscopy data in Fig. 1b (aggregation-stage

730 Dictyostelium dark green (dark green), HL-60 (dark red) and fish keratocyte (yellow)). (b-g) A time-series

731 sample of experimental data closest to the average morphology and the corresponding top-ranked

732 simulations; vegetative-stage Dictyostelium (b, c), nocodazole-treated HL-60 (d, e) Dicyostelium racE- (f,

733 g). (h) The top-ranked simulations (rank1 and rank2; magenta circles) for Dicyostelium (veg) shifted

734 toward Dictyostelium (agg) by shifting $a_{\mathrm{W}}$ from 2.4 to 4 (solid line), and toward the orange region by

735 shifting $W_{\text {tot }}$ from 80 to 95 (dotted line). 


\section{References}

739 1. L. Li, S. F. Nørrelykke, E. C. Cox, Persistent cell motion in the absence of external signals: a 740 search strategy for eukaryotic cells. PLOS ONE. 3, e2093 (2008).

741 2. M. Otsuji, Y. Terashima, S. Ishihara, S. Kuroda, K. Matsushima, A conceptual molecular network 742 for chemotactic behaviors characterized by feedback of molecules cycling between the membrane 743 and the cytosol. Sci Signal. 3, ra89-ra89 (2010).

744 3. X. Liu, E. S. Welf, J. M. Haugh, Linking morphodynamics and directional persistence of T 745 lymphocyte migration. J R Soc Interface. 12, 20141412-20141412 (2015).

746 4. M. F. Krummel, F. Bartumeus, A. Gérard, T cell migration, search strategies and mechanisms. Nat 747 Rev Immunol. 16, 193-201 (2016).

$748 \quad 5 . \quad$ C. M. Witt, S. Raychaudhuri, B. Schaefer, A. K. Chakraborty, E. A. Robey, Directed Migration of 749 Positively Selected Thymocytes Visualized in Real Time. PLoS Biol. 3, e160-8 (2005).

6. N. Andrew, R. H. Insall, Chemotaxis in shallow gradients is mediated independently of PtdIns 3kinase by biased choices between random protrusions. Nat Cell Biol. 9, 193-200 (2007).

7. L. Bosgraaf, P. J. M. Van Haastert, The ordered extension of pseudopodia by amoeboid cells in

8. A. Leithner et al., Diversified actin protrusions promote environmental exploration but are dispensable for locomotion of leukocytes. Nat Cell Biol. 18, 1253-1259 (2016).

9. L. K. Fritz-Laylin et al., Actin-based protrusions of migrating neutrophils are intrinsically lamellar and facilitate direction changes. Elife. 6, 437 (2017).

10. M. Bergert, S. D. Chandradoss, R. A. Desai, E. Paluch, Cell mechanics control rapid transitions between blebs and lamellipodia during migration. Proc Natl Acad Sci USA. 109, 14434-14439 (2012).

761 11. R. J. Petrie, A. D. Doyle, K. M. Yamada, Random versus directionally persistent cell migration.

$762 \quad$ Nat Rev Mol Cell Biol. 10, 538-549 (2009).

763 12. J. Lee, Insights into cell motility provided by the iterative use of mathematical modeling and experimentation. AIMS Biophysics. 5, 97-124 (2018).

765 13. Y. Asano et al., Keratocyte-like locomotion in amiB-null Dictyostelium cells. 59, 17-27 (2004).

766 14. Y. Miao et al., Altering the threshold of an excitable signal transduction network changes cell 767 migratory modes. Nat Cell Biol. 19, 329-340 (2017).

768 15. Z. Chen et al., Gleevec, an Abl Family Inhibitor, Produces a Profound Change in Cell Shape and Migration. PLoS ONE. 8, e52233-14 (2013). 
770 16. A. Van Keymeulen et al., To stabilize neutrophil polarity, PIP3 and Cdc42 augment RhoA activity 771 at the back as well as signals at the front. J Cell Biol. 174, 437-445 (2006).

772 17. H. Hattori et al., Small-molecule screen identifies reactive oxygen species as key regulators of 773 neutrophil chemotaxis. Proc Natl Acad Sci USA. 107, 3546-3551 (2010).

774 18. K. Keren et al., Mechanism of shape determination in motile cells. Nat. Cell. Biol. 453, 475-480 775 (2008).

776 19. X. Ma, O. Dagliyan, K. M. Hahn, G. Danuser, Profiling cellular morphodynamics by 777 spatiotemporal spectrum decomposition. PLoS Comput Biol. 14, e1006321 (2018).

778 20. L. Tweedy, B. Meier, J. Stephan, D. Heinrich, R. G. Endres, Distinct cell shapes determine 779 accurate chemotaxis. Sci. Rep. 3, 2606 (2013).

780 21. E. Alizadeh, S. M. Lyons, J. M. Castle, A. Prasad, Measuring systematic changes in invasive 781 cancer cell shape using Zernike moments. Integr Biol (Camb). 8, 1183-1193 (2016).

782 22. M. K. Driscoll, J. T. Fourkas, W. Losert, Local and global measures of shape dynamics. Phys Biol. $783 \mathbf{8}, 055001(2011)$.

784 23. R. M. Cooper, N. S. Wingreen, E. C. Cox, An excitable cortex and memory model successfully predicts new pseudopod dynamics. PLoS ONE. 7, e33528 (2012).

24. C. Bakal, J. Aach, G. Church, N. Perrimon, Quantitative morphological signatures define local signaling networks regulating cell morphology. Science. 316, 1753-1756 (2007).

788 25. Z. Yin et al., A screen for morphological complexity identifies regulators of switch-like transitions between discrete cell shapes. Nat. Cell. Biol. 15, 860-871 (2013).

790 26. G. Danuser, J. Allard, A. Mogilner, Mathematical modeling of eukaryotic cell migration: insights beyond experiments. Annu Rev Cell Dev Biol. 29, 501-528 (2013).

27. V. Te Boekhorst, L. Preziosi, P. Friedl, Plasticity of Cell Migration In Vivo and In Silico. Annu Rev Cell Dev Biol. 32, 491-526 (2016).

28. J. Lee, A. Ishihara, J. A. Theriot, K. Jacobson, Principles of locomotion for simple-shaped cells. Nat. Cell. Biol. 362, 167-171 (1993).

29. Y. Mori, A. Jilkine, L. Edelstein-Keshet, Wave-pinning and cell polarity from a bistable reactiondiffusion system. Biophys J. 94, 3684-3697 (2008).

30. P. J. M. Van Haastert, I. Keizer-Gunnink, A. Kortholt, Coupled excitable Ras and F-actin activation mediates spontaneous pseudopod formation and directed cell movement. Mol Biol Cell. 28, 922-934 (2017).

801 31. C. Arrieumerlou, T. Meyer, A Local Coupling Model and Compass Parameter for Eukaryotic Chemotaxis. Dev Cell. 8, 215-227 (2005). 
803 32. H. W. Yang, S. R. Collins, T. Meyer, Locally excitable Cdc42 signals steer cells during chemotaxis. Nat Cell Biol. 18, 191-201 (2015).

805 33. M. Tang et al., Evolutionarily conserved coupling of adaptive and excitable networks mediates

807 34. K. Plak et al., GxcC connects Rap and Rac signaling during Dictyostelium development. BMC $808 \quad$ Cell Biol. 14, 6 (2013).

809 35. S. Beco et al., Optogenetic dissection of Rac1 and Cdc42 gradient shaping. Nat Commun, 1-13 $810 \quad$ (2018).

811 36. H. Senoo et al., Phosphorylated Rho-GDP directly activates mTORC2 kinase towards AKT 812 through dimerization with Ras-GTP to regulate cell migration. Nat Cell Biol. 21, 867-878 (2019).

813 37. I. Niculescu, J. Textor, R. J. de Boer, Crawling and Gliding: A Computational Model for Shape814 Driven Cell Migration. PLoS Comput Biol. 11, e1004280 (2015).

815 38. S. Alonso, M. Stange, C. Beta, Modeling random crawling, membrane deformation and intracellular polarity of motile amoeboid cells. PLoS ONE. 13, e0201977 (2018).

817 39. W. R. Holmes, A. E. Carlsson, L. Edelstein-Keshet, Regimes of wave type patterning driven by refractory actin feedback: transition from static polarization to dynamic wave behaviour. Phys Biol. 9, 046005 (2012).

40. Y. Cao, E. Ghabache, W.-J. Rappel, Plasticity of cell migration resulting from mechanochemical coupling. Elife. 8, 107 (2019).

822 41. M. Edwards et al., Insight from the maximal activation of the signal transduction excitable network in Dictyostelium discoideum. Proc Natl Acad Sci USA. 115, E3722-E3730 (2018).

824 42. M. K. Driscoll et al., Cell shape dynamics: from waves to migration. PLoS Comput Biol. 8, 825 e1002392(2012).

826 43. H. Takagi, M. J. Sato, T. Yanagida, M. Ueda, PLOS ONE: Functional Analysis of Spontaneous Cell Movement under Different Physiological Conditions. PLoS ONE (2008).

828 44. J. Xu, F. Wang, A. Van Keymeulen, M. Rentel, Neutrophil microtubules suppress polarity and enhance directional migration. Proc Natl Acad Sci USA. 102, 6884-6889 (2005).

830 45. T. Y.-C. Tsai et al., Efficient Front-Rear Coupling in Neutrophil Chemotaxis by Dynamic Myosin

832 46. C. Litschko et al., Functional integrity of the contractile actin cortex is safeguarded by multiple Diaphanous-related formins. Proc Natl Acad Sci USA. 116, 3594-3603 (2019).

834 47. J. Satulovsky, R. Lui, Y.-L. Wang, Exploring the control circuit of cell migration by mathematical modeling. Biophys J. 94, 3671-3683 (2008). 
836 48. O. Nagel et al., Geometry-Driven Polarity in Motile Amoeboid Cells. PLoS ONE. 9, e113382 837 (2014).

838 49. S. Kumar et al., Cdc42 regulates neutrophil migration via crosstalk between WASp, CD11b, and

50. C.-H. Huang, M. Tang, C. Shi, P. A. Iglesias, P. N. Devreotes, An excitable signal integrator couples to an idling cytoskeletal oscillator to drive cell migration. Nat Cell Biol. 15, 1307-1316 (2013).

843 51. T. A. Burke et al., Homeostatic Actin Cytoskeleton Networks Are Regulated by Assembly Factor Competition for Monomers. Current Biology. 24, 579-585 (2014).

845 52. A. J. Lomakin et al., Competition for actin between two distinct F-actin networks defines a bistable switch for cell polarization. Nat. Cell. Biol. 17, 1435-1445 (2015).

847 53. C. Suarez, D. R. Kovar, Internetwork competition for monomers governs actin cytoskeleton organization. Nat Rev Mol Cell Biol. 17, 799-810 (2016).

54. A. J. Davidson, C. Amato, P. A. Thomason, R. H. Insall, WASP family proteins and formins compete in pseudopod- and bleb-based migration. J Cell Biol. 217, 701-714 (2018).

851 55. J. Bush, K. Franek, J. Cardelli, Cloning and characterization of seven novel Dictyostelium 852 discoideum rac-related genes belonging to the rho family of GTPases. Gene. 136, 61-68 (1993).

853 56. J. E. Bear, J. F. Rawls, C. L. Saxe, SCAR, a WASP-related Protein, Isolated as a Suppressor of

855 57. P. Maiuri et al., Actin Flows Mediate a Universal Coupling between Cell Speed and Cell Persistence. Cell. 161, 374-386 (2015).

857 58. L. Yolland et al., Persistent and polarized global actin flow is essential for directionality during cell migration. Nat. Cell. Biol., 1-27 (2019).

59. Y. Fukui, T. Kitanishi-Yumura, S. Yumura, Myosin II-independent F-actin flow contributes to cell locomotion in dictyostelium. J Cell Sci. 112 ( Pt 6), 877-886 (1999).

861 60. B. R. Graziano et al., Cell confinement reveals a branched-actin independent circuit for neutrophil polarity. PLoS Biol. 17, e3000457-34 (2019).

61. F. Knoch, M. Tarantola, E. Bodenschatz, W.-J. Rappel, Modeling self-organized spatio-temporal patterns of PIP3 and PTEN during spontaneous cell polarization. Phys Biol. 11, 046002 (2014).

865 62. D. Shao, W.-J. Rappel, H. Levine, Computational model for cell morphodynamics. Phys Rev Lett. 105, 108104 (2010). 
870 64. E. Tunnacliffe, A. M. Corrigan, J. R. Chubb, Promoter-mediated diversification of transcriptional

871 bursting dynamics following gene duplication. Proc Natl Acad Sci USA. 115, 8364-8369 (2018).

872 65. T. Gregor, K. Fujimoto, N. Masaki, S. Sawai, The onset of collective behavior in social amoebae.

$873 \quad$ Science. 328, 1021-1025 (2010).

874 66. C. Okimura, A. Taniguchi, S. Nonaka, Y. Iwadate, Rotation of stress fibers as a single wheel in

875 migrating fish keratocytes. Sci. Rep. 8, 1-10 (2018).

876 67. D. Taniguchi et al., Phase geometries of two-dimensional excitable waves govern self-organized

877 morphodynamics of amoeboid cells. Proc Natl Acad Sci USA. 110, 5016-5021 (2013).

878 68. R. Winklbauer, Cell adhesion strength from cortical tension - an integration of concepts. $J$ Cell $879 \quad$ Sci. 128, 3687-3693 (2015).

880 69. M. J. Footers, J. W. J. Kerssemakers, J. A. Theriot, M. Dogterom, Direct measurement of force generation by actin filament polymerization using an optical trap. Proc. Natl. Acad. Sci. USA. 104, 2181-2186 (2007).

70. M. P. Clausen, H. Colin-York, F. Schneider, C. Eggeling, M. Fritzsche, Dissecting the actin cortex density and membrane- cortex distance in living cells by super-resolution microscopy. $J$ Phys $D$ Appl Phys. 50, 064002 (2017).

71. S. Fukushima, S. Matsuoka, M. Ueda, Excitable dynamics of Ras triggers spontaneous symmetry breaking of PIP3 signaling in motile cells. J Cell Sci. 132, jcs224121 (2019).

72. A. T. Sasaki et al., G protein-independent Ras/PI3K/F-actin circuit regulates basic cell motility. $J$ Cell Biol. 178, 185-191 (2007).

73. M.-J. Wang, Y. Artemenko, W.-J. Cai, P. A. Iglesias, P. N. Devreotes, The Directional Response of Chemotactic Cells Depends on a Balance between Cytoskeletal Architecture and the External Gradient. Cell Rep. 9, 1110-1121 (2014). 
Fig.1

(a)

(b)

(c)

bioRxiv preprint doi: https://doi.org/10.1101/2020.09.06.285411; this version posted September 8, 2020. The copyright holder for this preprint

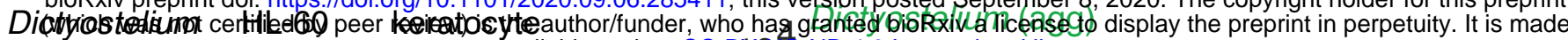
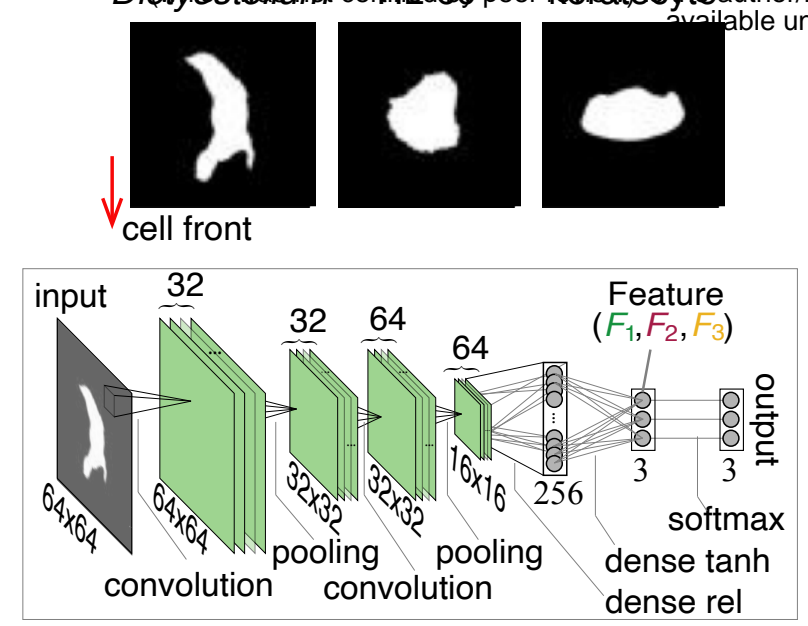
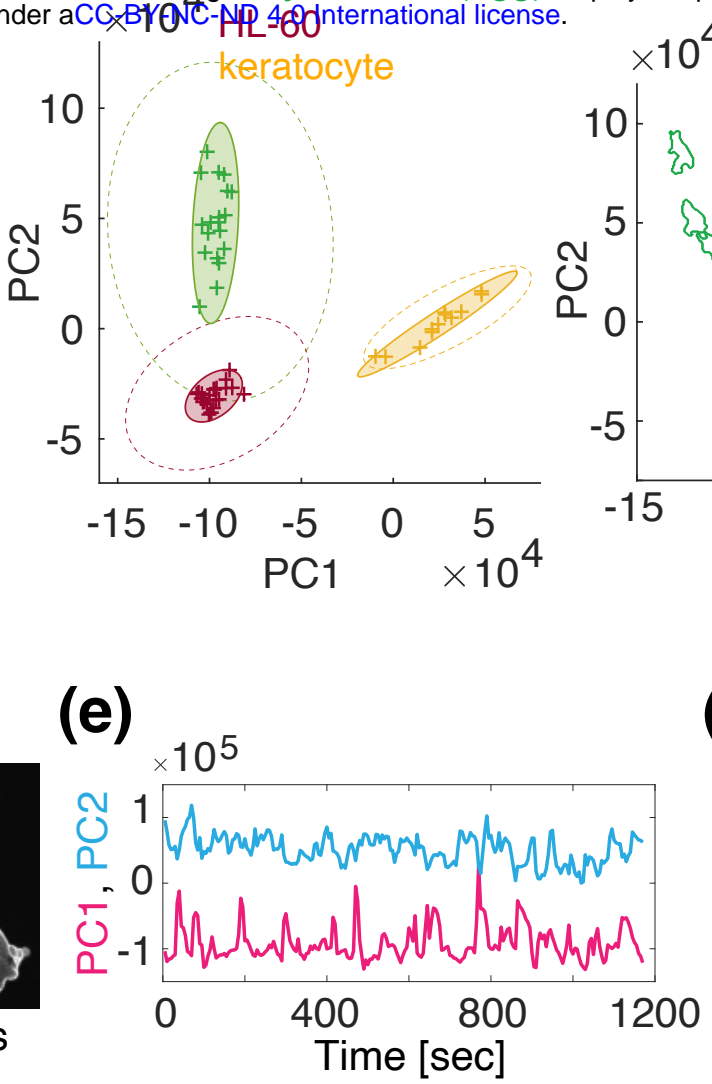

(e)

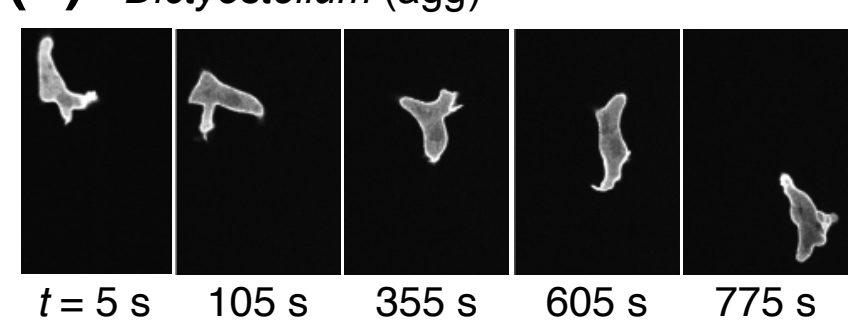

(d) Dictyostelium (agg)

(g)

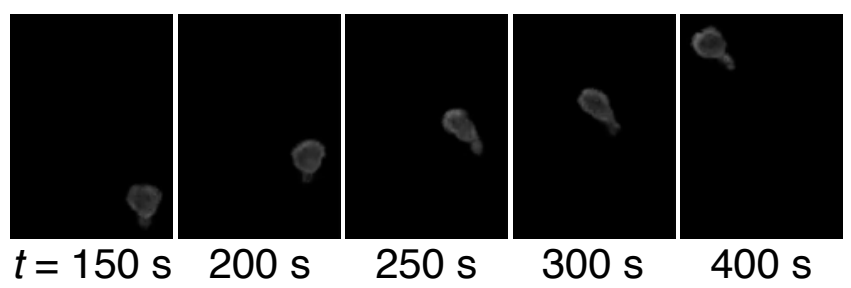

(j) keratocyte
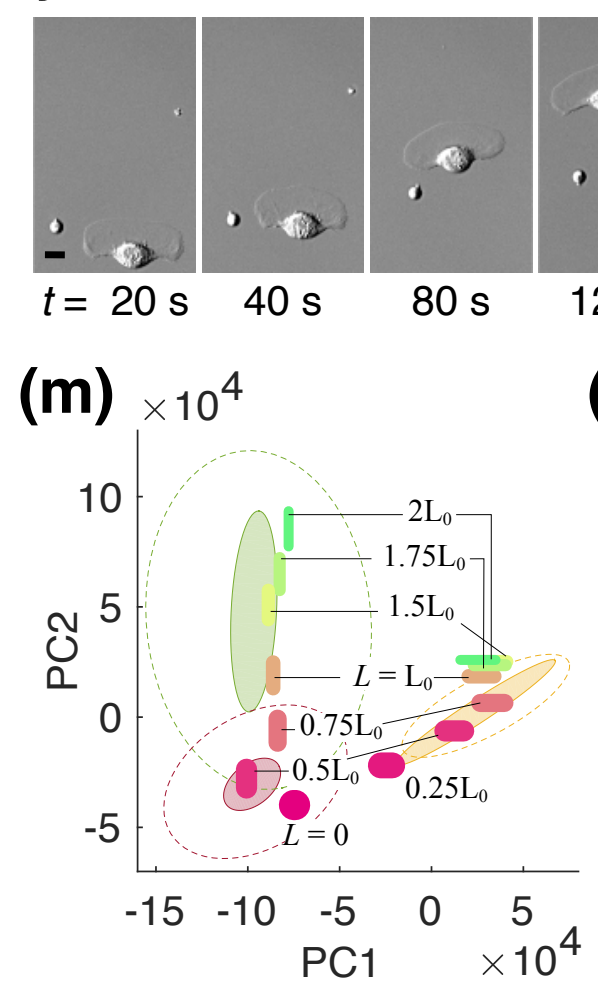

(h)

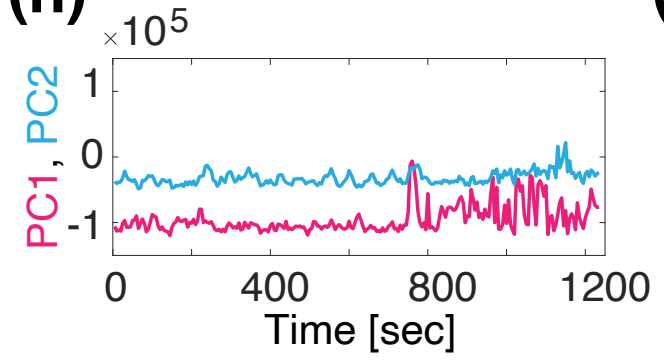

(f) $\times 10^{4}$

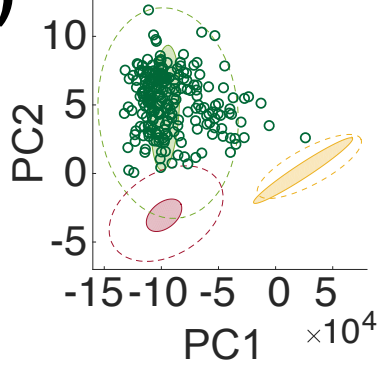

(i) 10

ญ 5

$-5$

$-15-10-5 \quad 0 \quad 5$
PC1 $\times 10^{4}$

(I) 10

\begin{tabular}{ll}
\multirow{2}{}{} & 5 \\
\hdashline & 0
\end{tabular}

$-5$

$-15-10-5 \quad 0 \quad 5$

PC1 $\times 10^{4}$
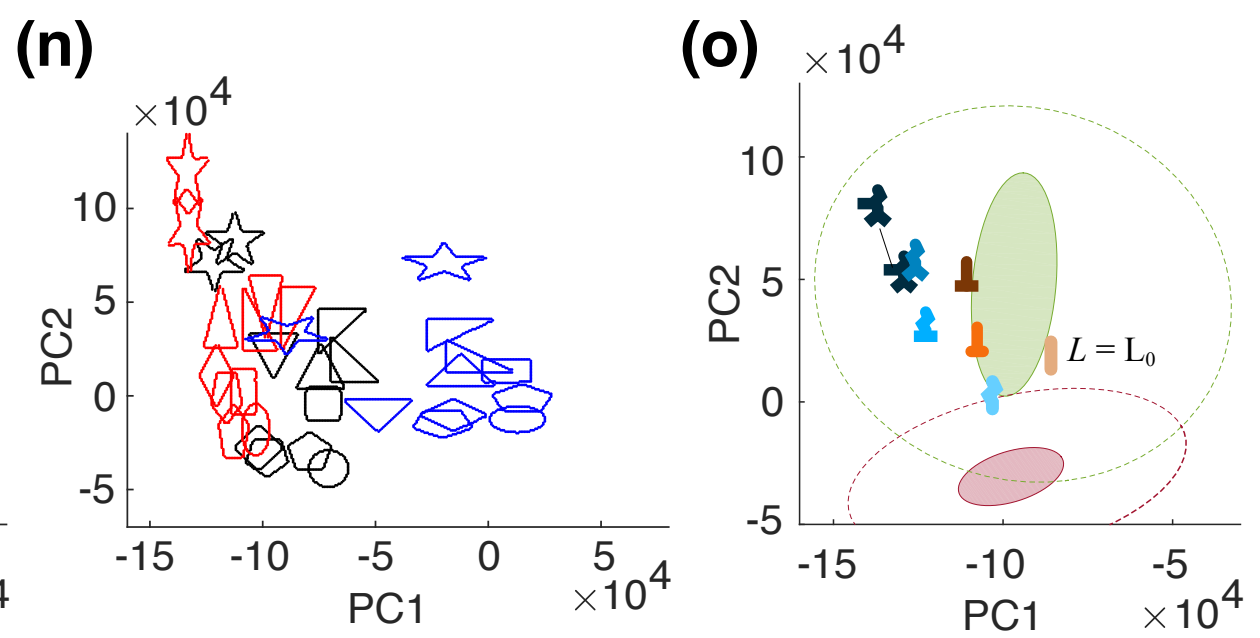
Fig.2

(a)

(b) $V, U, W$

Rear<smiles>[Y10]C1CCC([Y])CC1</smiles>

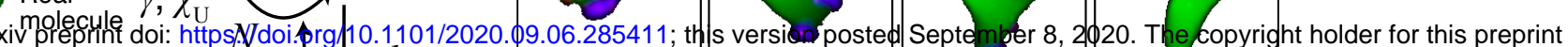
w wals/not certified by peer review)/ss, the author/funder, who thas granted bioR xiv a license to display the preprint in perpetuity. It is made

Front

molecules

available under $66^{2} 0^{-B Y}$

$700 \mathrm{~s}$ 't $740 \mathrm{~s}$

migration

direction
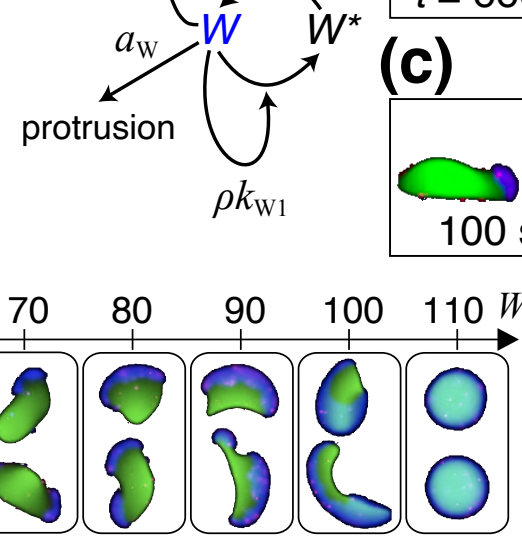

(d)
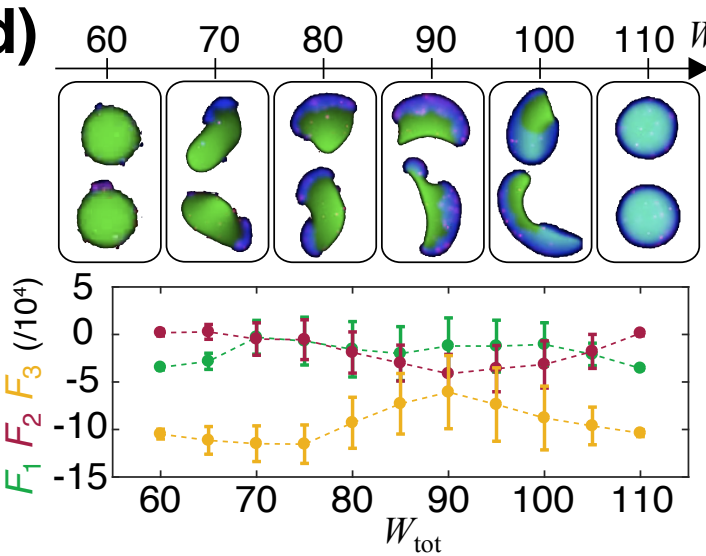

(f)

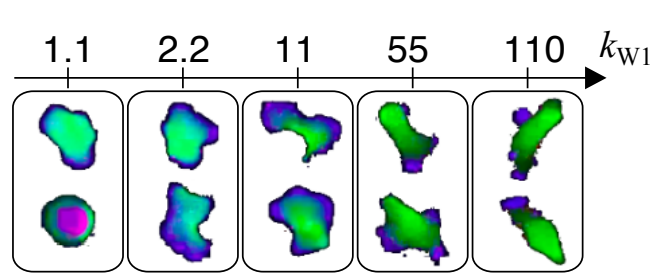

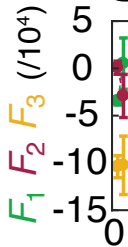

(h)
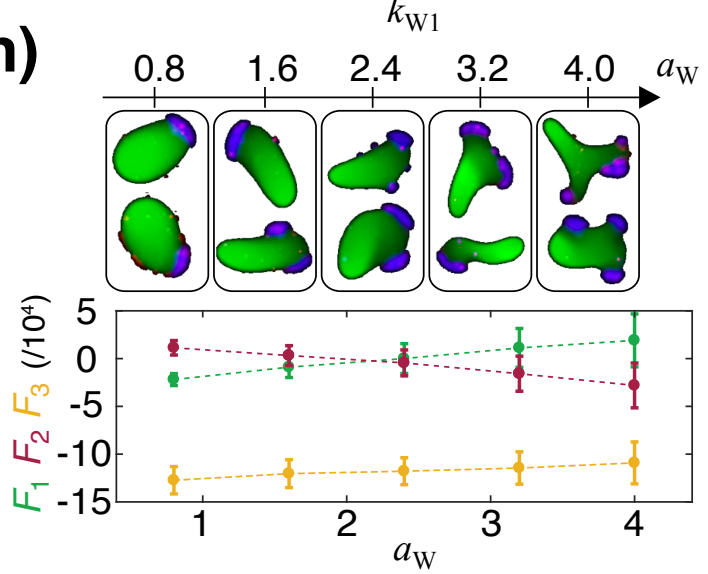

(j)

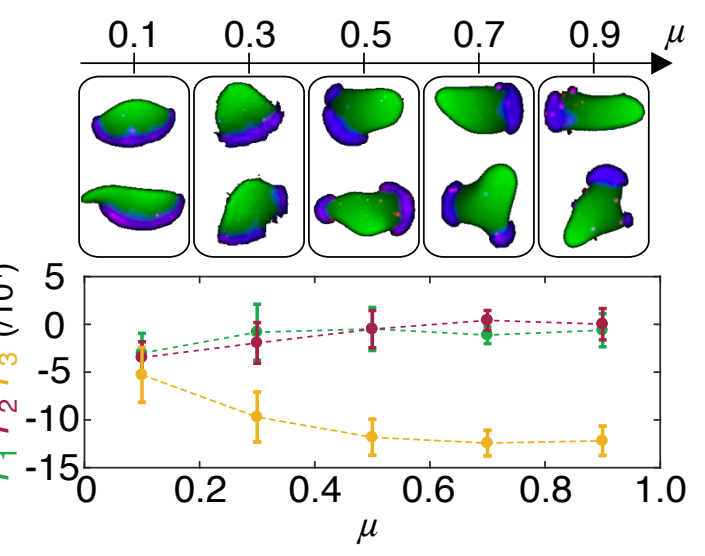

(k)

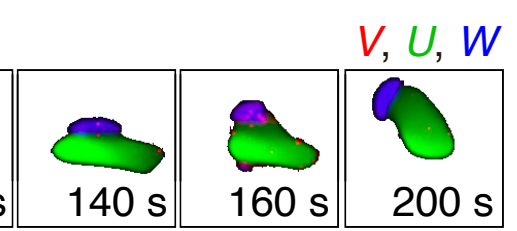

(e)

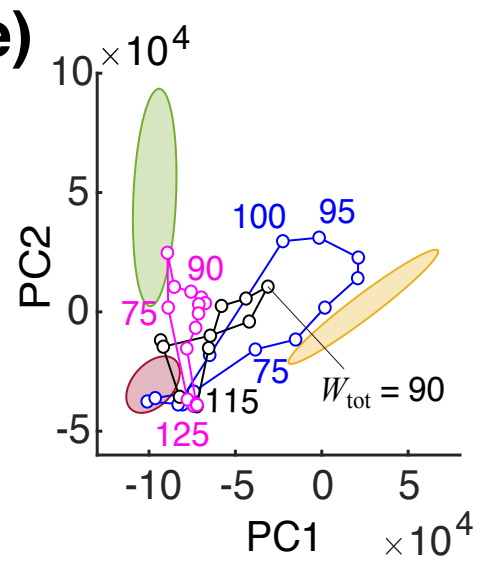

(g)

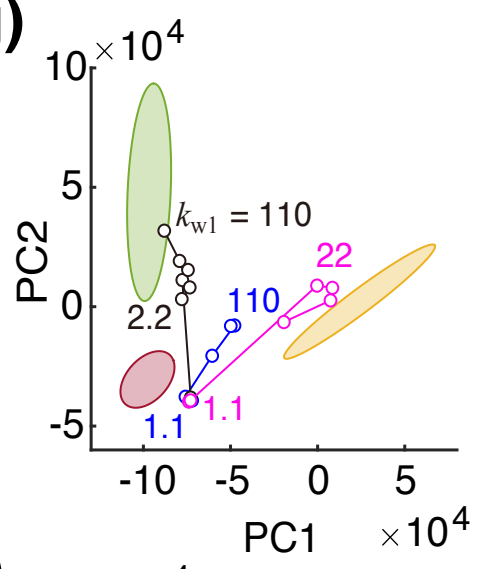

(i)
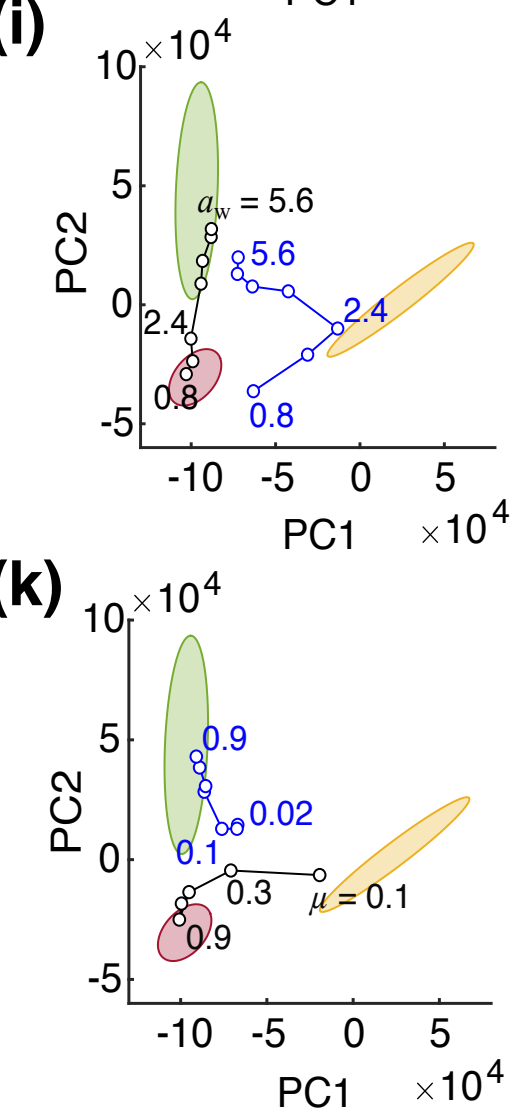


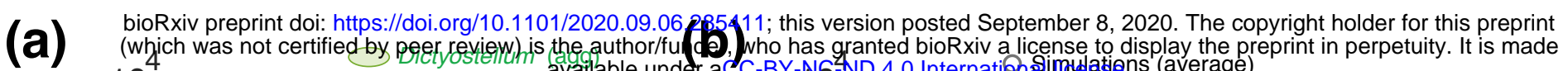
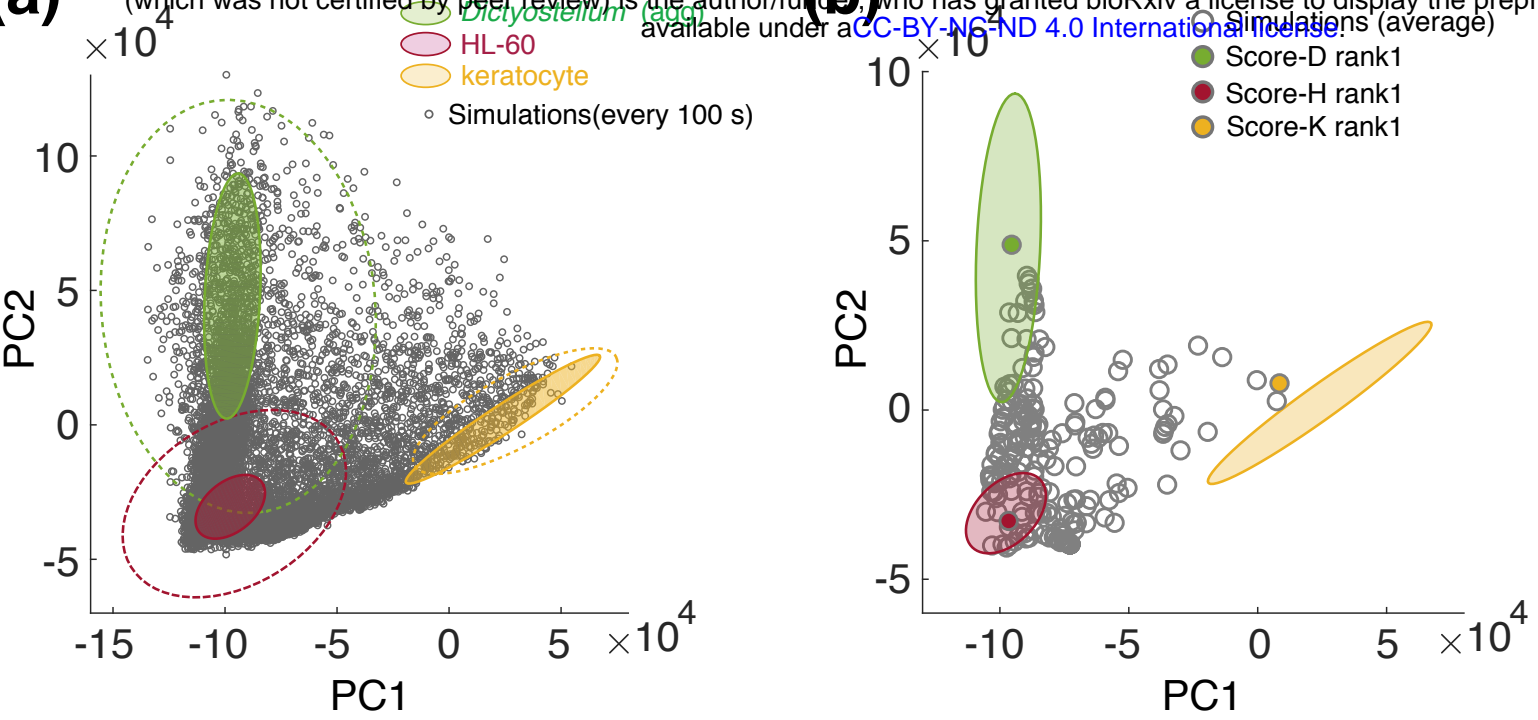

(c)

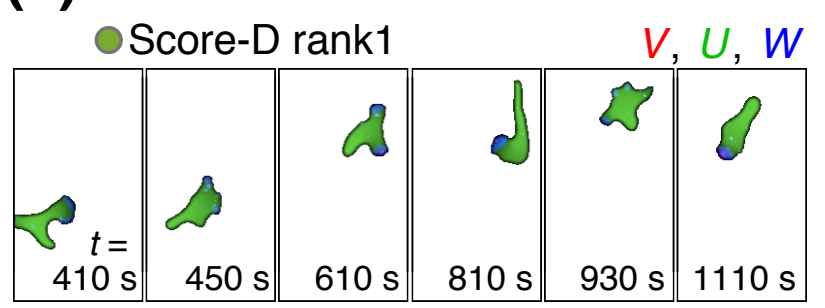

(f)

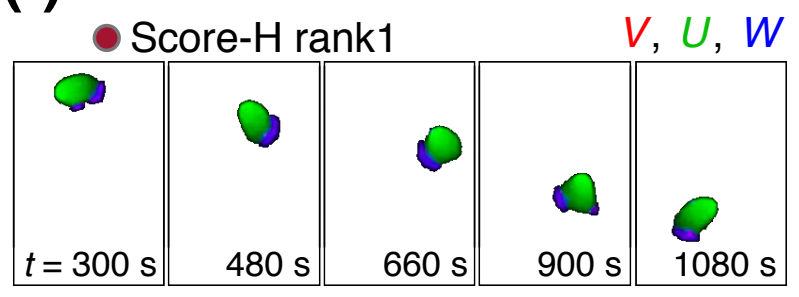

(i)

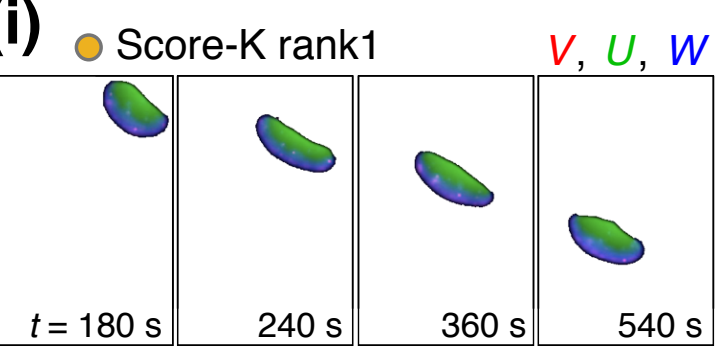

(d)

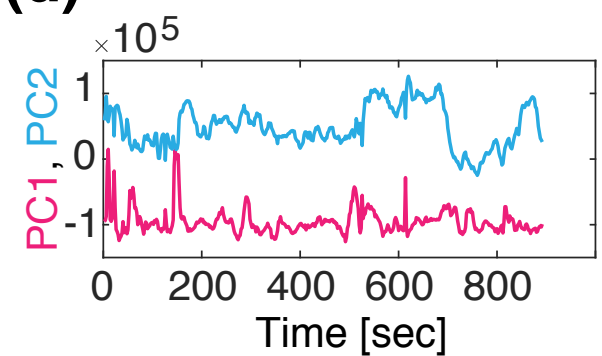

(g)

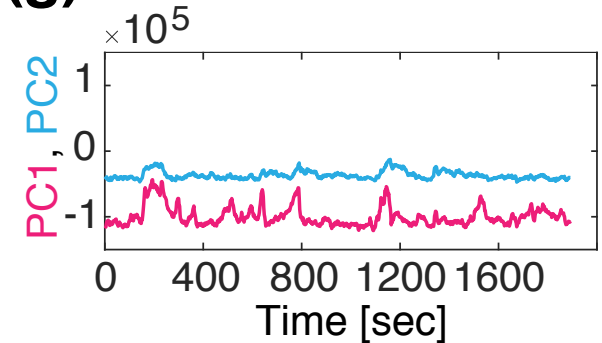

(j)

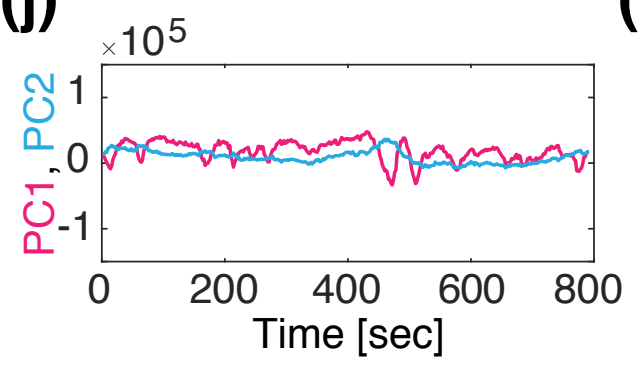

(e)

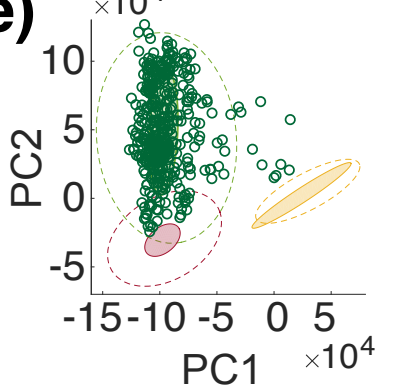

(h) $\times 10^{4}$

ญ 5

$-5$

$-15-10-5 \quad 05$
PC1 $\times 10^{4}$

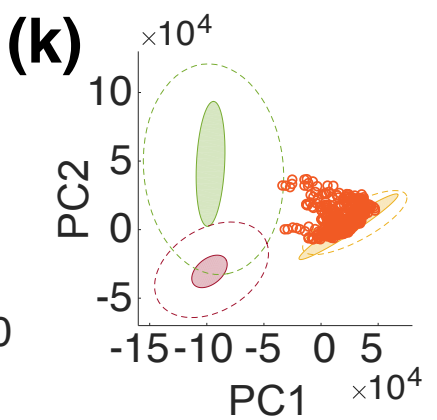


(a)

Dictyostelium (agg)

Rank1 simulations

(b)

HL-60

Rank1 simulations

which was not doi: https://doi.org/10.1101/2020.09.06.285411; this Yersigmpdsted September 8, 2020.The copyright holder for this preprint 15 을 2elongation

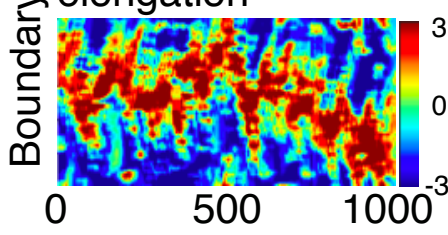

Time [sec]

(c)

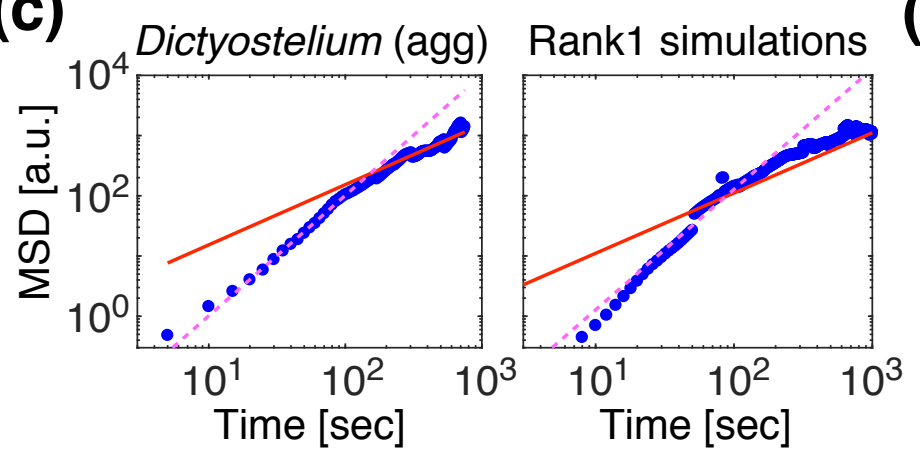

(e)
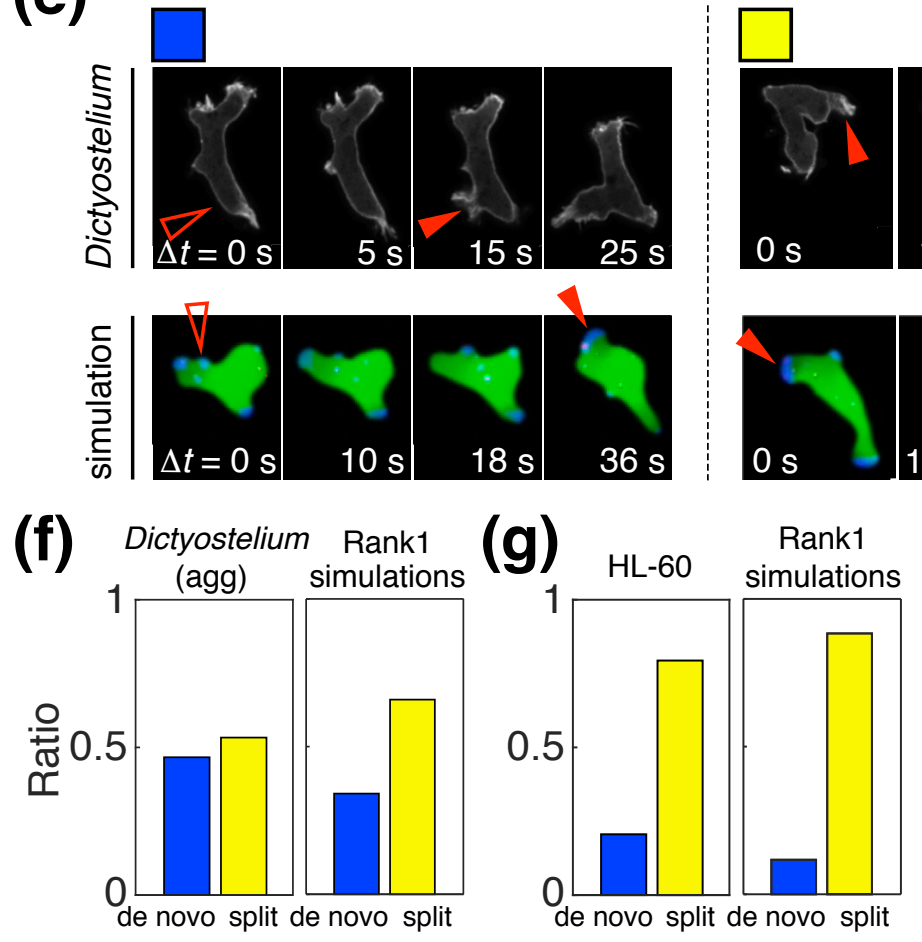

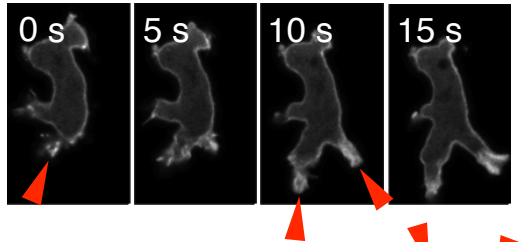

(d)

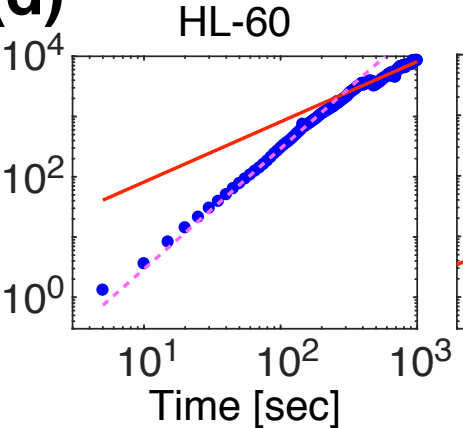

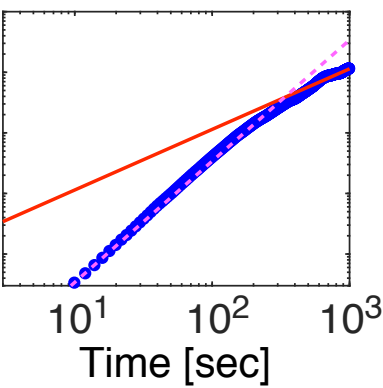

Rank1 simulations

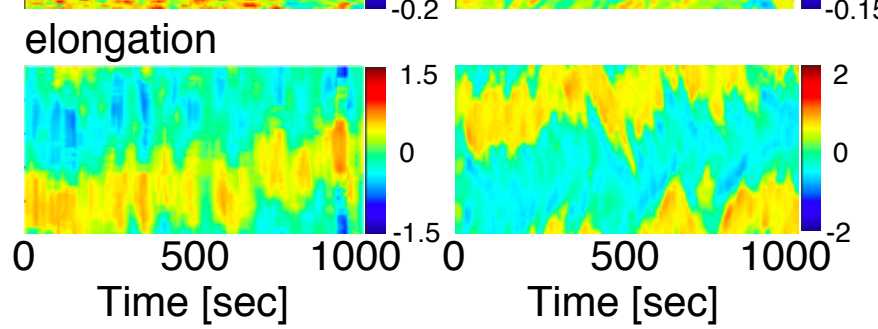

15

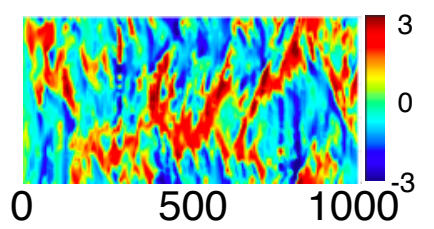

Time [sec]
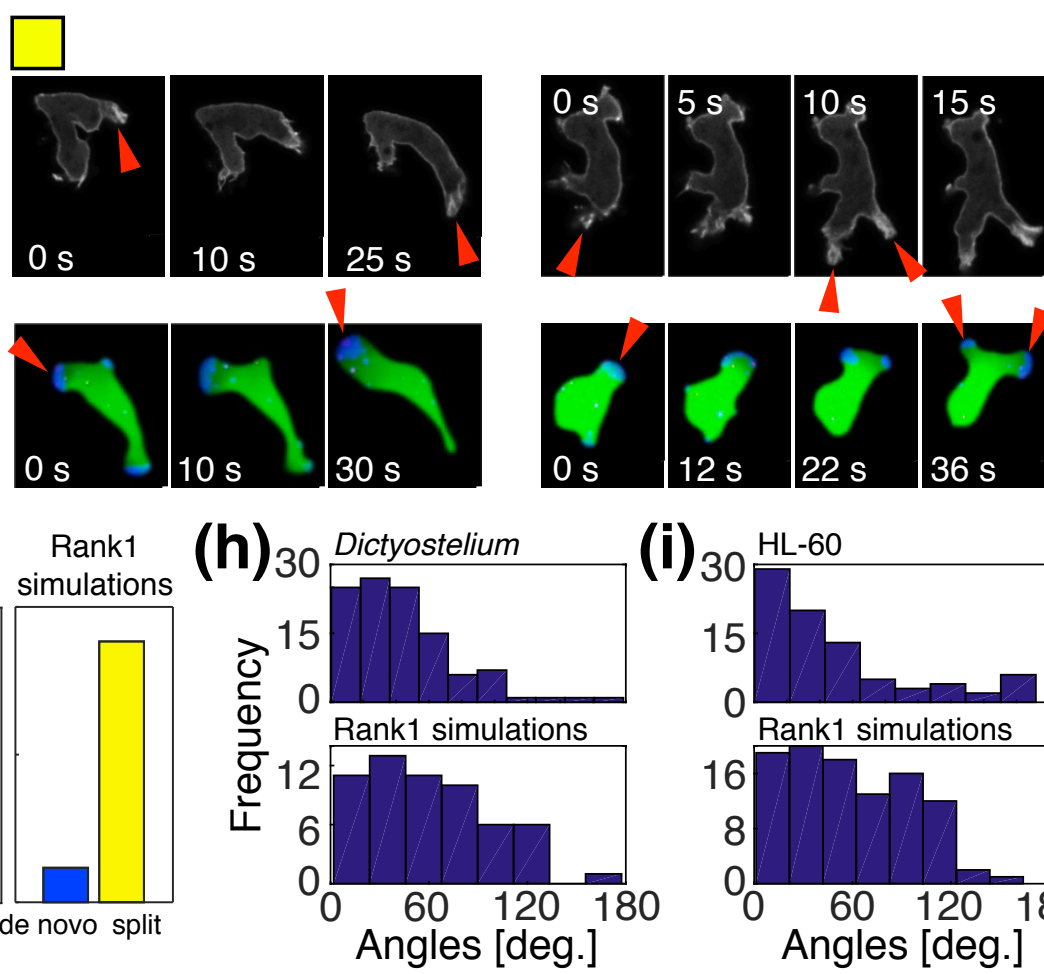

(h) 30 Dictyostelium

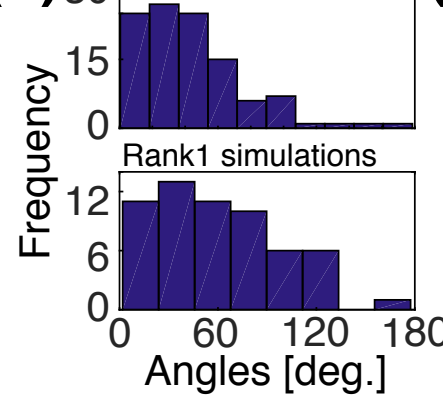

(i) $30 \stackrel{\mathrm{HL}-60}{ }$

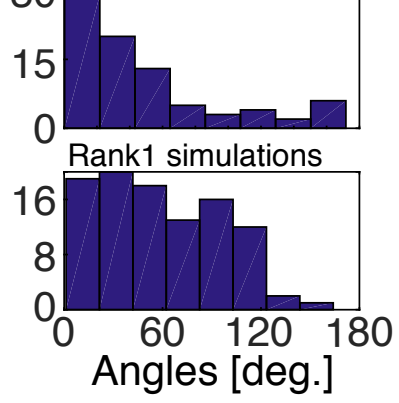



(a) $10^{\times 10^{4}}$
bloRxiv preprint doi: https://doi.org/10.1101/2020.09.06.285411; this version posted September 8, 2020. The copyright holder for this preprint

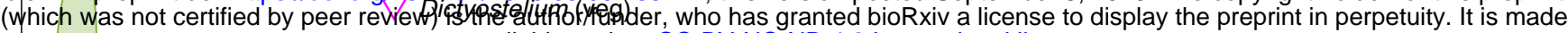

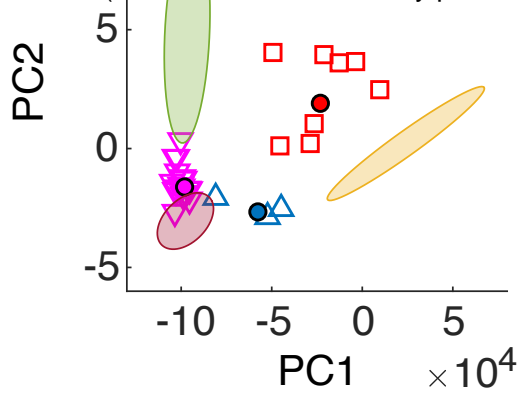
$\triangle \mathrm{HL}-60$ (nocodazole)
Score-D(veg) rank1
Score-D(racE-) rank1
Score-H(noco) rank1

(b) Dictyostelium (veg)

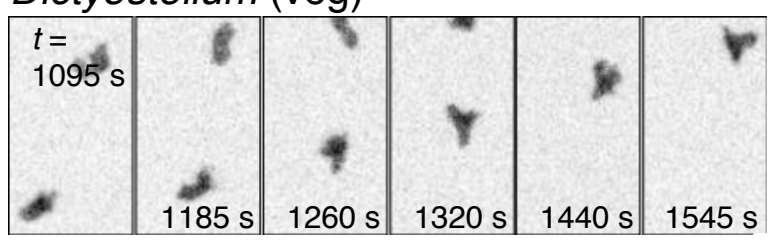

(c) Score-D(veg) rank1 $\quad V, U, W$

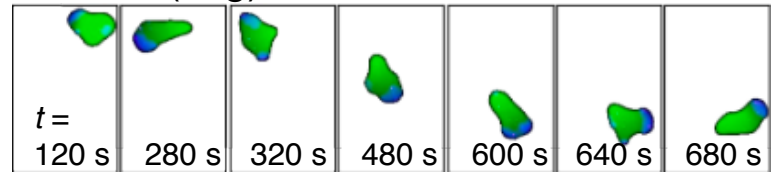

(d) $\mathrm{HL}-60$ (nocodazole)

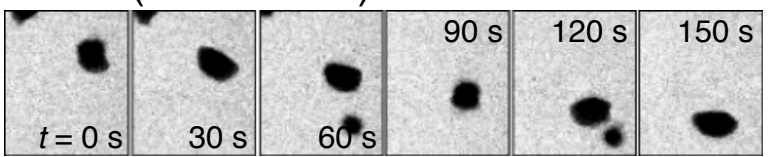

(e) Score-H(noco) rank1

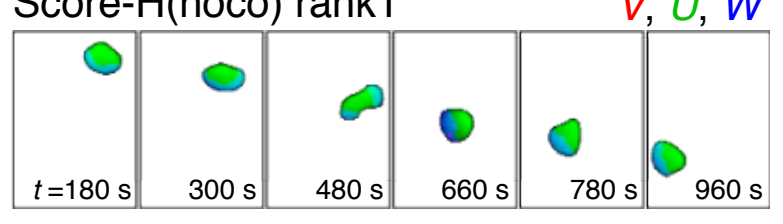

(f) Dictyostelium (racE-)

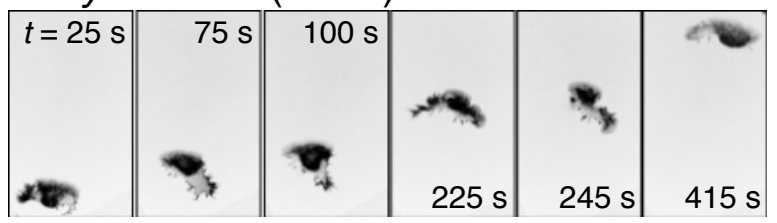

(g) Score- $D$ (racE-) rank1

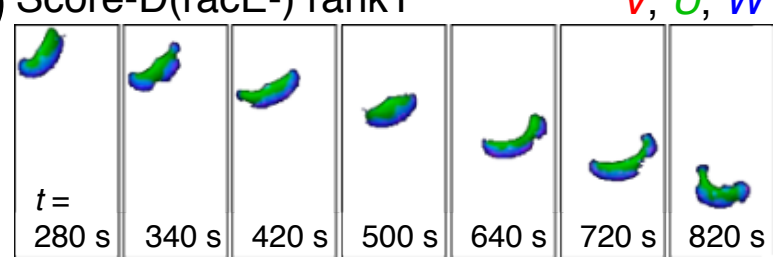

(h)

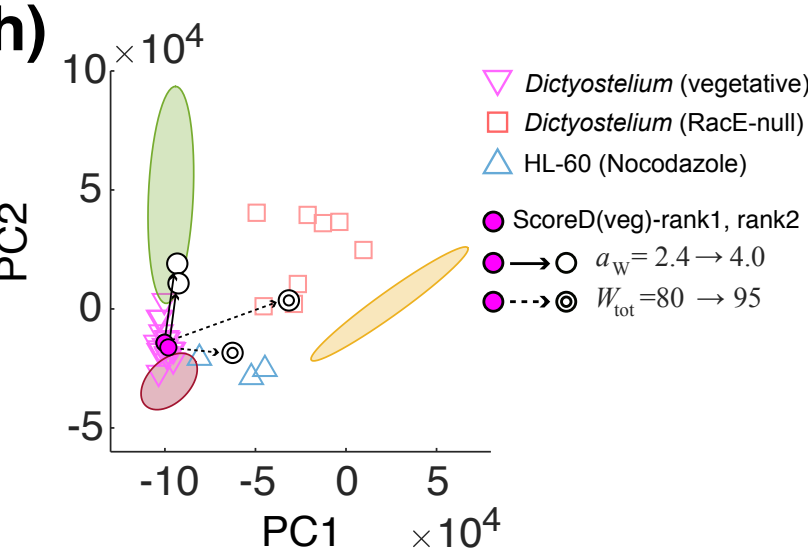

